\title{
Multisymplectic and Polysymplectic Structures on Fiber Bundles *
}

\author{
Michael Forger and Leandro Gomes ${ }^{\dagger}$ \\ Departamento de Matemática Aplicada \\ Instituto de Matemática e Estatística \\ Universidade de São Paulo \\ BR-05315-970 São Paulo, S.P., Brazil
}

\begin{abstract}
We introduce the concepts of a multisymplectic structure and a polysymplectic structure on a general fiber bundle over a general base manifold, define the concept of the symbol of a multisymplectic form, which is a polysymplectic form representing its leading order contribution, and prove Darboux theorems for the existence of canonical local coordinates.
\end{abstract}

Universidade de São Paulo

RT-MAP-0702

December 2007

\footnotetext{
${ }^{*}$ Work partially supported by CAPES (Coordenação de Aperfeiçoamento de Pessoal de Nível Superior) and CNPq (Conselho Nacional de Desenvolvimento Científico e Tecnológico), Brazil

$\dagger$ Work done in partial fulfillment of the requirements for the degree of Doctor in Science
} 


\section{Introduction}

Multisymplectic geometry is increasingly recognized as providing the appropriate mathematical framework for classical field theory from the hamiltonian point of view - just as symplectic geometry does for classical mechanics. Unfortunately, the development of this new area of differential geometry has for a long time been hampered by the lack of a fully satisfactory definition of the concept of a multisymplectic structure, which should be mathematically simple as well as in harmony with the needs of applications to physics; the same goes for the closely related notion of a polysymplectic structure.

The main goal of this paper is to provide such a definition and establish a general relation between the two types of structure.

To set the stage, let us consider a simple analogy. The symplectic forms encountered in classical mechanics can locally all be written in the form

$$
\omega=d q^{i} \wedge d p_{i}
$$

where $q^{1}, \ldots, q^{N}, p_{1}, \ldots, p_{N}$ are a particular kind of local coordinates on phase space known as canonical coordinates or Darboux coordinates. Introducing time $t$ and energy $E$ as additional variables (which is essential, e.g., for incorporating non-autonomous systems into the symplectic framework of hamiltonian mechanics), this equation is replaced by

$$
\omega=d q^{i} \wedge d p_{i}+d E \wedge d t
$$

where $t, q^{1}, \ldots, q^{N}, p_{1}, \ldots, p_{N}, E$ can be viewed as canonical coordinates on an extended phase space. Similarly, the multisymplectic forms encountered in classical field theory over an $n$-dimensional space-time manifold $M$ can locally all be written in the form

$$
\omega=d q^{i} \wedge d p_{i}^{\mu} \wedge d^{n} x_{\mu}-d p \wedge d^{n} x,
$$

where $x^{\mu}, q^{i}, p_{i}^{\mu}, p(1 \leqslant \mu \leqslant n, 1 \leqslant i \leqslant N)$ can again be viewed as canonical coordinates on some extended multiphase space. Here, the $x^{\mu}$ are (local) coordinates for $M$, while $p$ is still a single energy variable (except for a sign), $d^{n} x$ is the (local) volume form induced by the $x^{\mu}$ and $d^{n} x_{\mu}$ is the (local) $(n-1)$-form obtained by contracting $d^{n} x$ with $\partial_{\mu} \equiv \partial / \partial x^{\mu}$ :

$$
d^{n} x_{\mu}=\mathrm{i}_{\partial_{\mu}} d^{n} x
$$

The idea of introducing "multimomentum variables" labelled by an additional spacetime index $\mu$ ( $n$ multimomentum variables $p_{i}^{\mu}$ for each position variable $q^{i}$ ) goes back to the work of de Donder [1] and Weyl [2] in the 1930's (and perhaps even further) and has been recognized ever since as being an essential and unavoidable ingredient in any approach to a generally covariant hamiltonian formulation of classical field theory. Understanding the proper geometric setting for this kind of structure, however, has baffled both mathematicians and physicists for decades, as witnessed by the large number of different proposals for an appropriate global framework that can be found in the literature. 
The by now standard example of a globally defined multisymplectic structure starts out from an arbitrary fiber bundle $E$ over $M$ called the configuration bundle (since its sections are the basic fields of the field theory under consideration) and whose typical fiber is an $N$-dimensional manifold $Q$ representing the configuration space, as in mechanics. Following Refs [3,4], for example, consider the vector bundle $\bigwedge_{1}^{n} T^{*} E$ of $n$-forms over $E$ that are $(n-1)$-horizontal (i.e., that vanish whenever contracted with more than 1 vertical vector field), with projection onto $E$ denoted by $\pi_{1}^{n}$ : its total space carries a naturally defined $n$-form $\theta$ which we shall refer to as the multicanonical form, given by

$$
\theta_{\alpha}\left(v_{1}, \ldots, v_{n}\right)=\alpha\left(T_{\alpha} \pi_{1}^{n} \cdot v_{1}, \ldots, T_{\alpha} \pi_{1}^{n} \cdot v_{n}\right)
$$

for $\alpha \in \bigwedge_{1}^{n} T^{*} E$ and $v_{1}, \ldots, v_{n} \in T_{\alpha}\left(\bigwedge_{1}^{n} T^{*} E\right)$, and which gives rise to a closed $(n+1)$ form $\omega=-d \theta$ : this is the multisymplectic form for the hamiltonian formalism of classical field theory. The construction can be easily extended to the more general situation of the vector bundle $\bigwedge_{r-1}^{k} T^{*} E$ of $k$-forms over $E$ that are $(k+1-r)$-horizontal (i.e., that vanish whenever contracted with more than $r-1$ vertical vector fields), with projection onto $E$ denoted by $\pi_{r-1}^{k}$, where $1 \leqslant r \leqslant k$ and $k+1-r \leqslant n$ : its total space carries a naturally defined $k$-form $\theta$ which we shall again refer to as the multicanonical form, given by

$$
\theta_{\alpha}\left(v_{1}, \ldots, v_{k}\right)=\alpha\left(T_{\alpha} \pi_{r-1}^{k} \cdot v_{1}, \ldots, T_{\alpha} \pi_{r-1}^{k} \cdot v_{k}\right)
$$

for $\alpha \in \bigwedge_{r-1}^{k} T^{*} E$ and $v_{1}, \ldots, v_{k} \in T_{\alpha}\left(\bigwedge_{r-1}^{k} T^{*} E\right)$, and which gives rise to a closed $(k+1)$ form $\omega=-d \theta$ : we propose to call it a multilagrangian form. This term is motivated by the observation that the vertical bundle for the projection $\pi_{r-1}^{k}$ onto $E$ is a distinguished lagrangian (maximal isotropic) subbundle of the tangent bundle of $\bigwedge_{r-1}^{k} T^{*} E$, and the existence of such a "multilagrangian subbundle" will turn out to play a central role in our general definition of multisymplectic and multilagrangian structures.

The standard local coordinate expressions can be obtained by starting out from local coordinates $\left(x^{\mu}, q^{i}\right)$ for $E$ composed of local coordinates $x^{\mu}$ for $M$ and local coordinates $q^{i}$ for $Q$ together with a local trivialization of $E$ over $M$ : these give rise to canonical local coordinates $\left(x^{\mu}, q^{i}, p_{i}^{\mu}, p\right)$ for $\bigwedge_{1}^{n} T^{*} E$ in which

$$
\theta=p_{i}^{\mu} d q^{i} \wedge d^{n} x_{\mu}+p d^{n} x
$$

So

$$
\omega=d q^{i} \wedge d p_{i}^{\mu} \wedge d^{n} x_{\mu}-d p \wedge d^{n} x,
$$

and more generally, to canonical local coordinates $\left(x^{\mu}, q^{i}, p_{i_{1} \ldots i_{s} ; \mu_{1} \ldots \mu_{k-s}}\right)(0 \leqslant s \leqslant r-1)$ for $\bigwedge_{r-1}^{k} T^{*} E$ in which

$$
\theta=\sum_{s=0}^{r-1} \frac{1}{s !} \frac{1}{(k-s) !} p_{i_{1} \ldots i_{s} ; \mu_{1} \ldots \mu_{k-s}} d q^{i_{1}} \wedge \ldots \wedge d q^{i_{s}} \wedge d x^{\mu_{1}} \wedge \ldots \wedge d x^{\mu_{k-s}},
$$

SO

$$
\omega=-\sum_{s=0}^{r-1} \frac{1}{s !} \frac{1}{(k-s) !} d p_{i_{1} \ldots i_{s} ; \mu_{1} \ldots \mu_{k-s}} \wedge d q^{i_{1}} \wedge \ldots \wedge d q^{i_{s}} \wedge d x^{\mu_{1}} \wedge \ldots \wedge d x^{\mu_{k-s}}
$$


In a more general context, when $\bigwedge_{r-1}^{k} T^{*} E$ is replaced by a manifold $P$ which is only supposed to be the total space of a fiber bundle over a base manifold $M$, Darboux's theorem guarantees the existence of canonical local coordinates in which $\omega$ is given by the expression in equation (9), under appropriate conditions on the form $\omega$. The central question is to figure out what precisely are these conditions.

A naive first guess would be to simply require the form $\omega$ to be closed and nondegenerate. However, unlike in the symplectic case, these conditions alone are far too weak to guarantee the validity of a Darboux theorem, even at the purely algebraic level. For certain purposes, they may be sufficient to derive results that are of interest (for an example, see Refs [5-7]), but this version of the definition of a multisymplectic structure even though often adopted in the literature, mostly for lack of a better one - is clearly inadequate. What is needed is an additional algebraic condition.

An indication of what should be this additional algebraic condition can be found in Ref. [8], but the Darboux theorem proved there covers a special situation which is disjoint from the case of interest for the applications to physics because the structure of the underlying manifold as the total space of a fiber bundle over space-time and the corresponding horizontality conditions are completely ignored. More specifically, Ref. [8] deals with what we call a multilagrangian form $\omega$ on a manifold, viewed as the total space of a fiber bundle whose base manifold $M$ is reduced to a point, so that the pertinent horizontality condition becomes empty and the corresponding expression for $\omega$ in canonical local coordinates takes the following form, analogous to equation (9):

$$
\omega=-\frac{1}{k !} d p_{i_{1} \ldots i_{k}} \wedge d q^{i_{1}} \wedge \ldots \wedge d q^{i_{k}}
$$

It is true that this generalizes the concept of a symplectic form to forms of higher degree, but in a direction that is far away from the concept of a multisymplectic form as encountered in classical field theory, and this discrepancy, which was clearly stated only much later [9] (see also Ref. [10], for example), has created a great deal of confusion in the literature. Taking into account that the term "multisymplectic" is already occupied at least since the mid 1970's [11-13], we believe its use in a quite different context such as that of Refs $[8,9]$ to be inappropriate and propose to correct this historical misnomer, replacing the term "multisymplectic" in that context by the term "multilagrangian".

In this paper, we shall follow a different approach, based on a new and more profound understanding of the link between multisymplectic and polysymplectic structures.

Polysymplectic structures in the hamiltonian approach to classical field theory seem to have been introduced in Ref. [14] and have been further investigated in Ref. [15] (where they were called " $k$-symplectic structures" - a terminology that we shall not follow in order not to increase the already existing confusion). Roughly speaking, polysymplectic forms are vector-valued analogues of symplectic forms. Similarly, the polylagrangian forms to be introduced in this paper are vector-valued analogues of the forms studied in Ref. [8].

The standard example of a globally defined polysymplectic structure is the one on the bundle $T^{*} E \otimes \hat{T}$ of $\hat{T}$-valued 1 -forms over a manifold $E$, with projection onto $E$ denoted 
by $\pi^{1}$, where $\hat{T}$ is a fixed finite-dimensional auxiliary vector space: its total space carries a naturally defined $\hat{T}$-valued 1 -form $\theta$ which we shall refer to as the polycanonical form, given by

$$
\theta_{\alpha}(v)=\alpha\left(T_{\alpha} \pi^{1} \cdot v\right)
$$

for $\alpha \in T^{*} E \otimes \hat{T}$ and $v \in T_{\alpha}\left(T^{*} E \otimes \hat{T}\right)$, and which gives rise to a closed $\hat{T}$-valued 2-form $\omega=-d \theta$ : this is the type of form called polysymplectic, for instance, in Ref. [14]. Again, the construction can be easily extended to the more general situation of the vector bundle $\bigwedge^{k} T^{*} E \otimes \hat{T}$ of $\hat{T}$-valued $k$-forms over $E$, with projection onto $E$ denoted by $\pi^{k}$ : its total space carries a naturally defined $\hat{T}$-valued $k$-form $\theta$ which we shall again refer to as the polycanonical form, given by

$$
\theta_{\alpha}\left(v_{1}, \ldots, v_{k}\right)=\alpha\left(T_{\alpha} \pi^{k} \cdot v_{1}, \ldots, T_{\alpha} \pi^{k} \cdot v_{k}\right)
$$

for $\alpha \in \bigwedge^{k} T^{*} E \otimes \hat{T}$ and $v_{1}, \ldots, v_{k} \in T_{\alpha}\left(\bigwedge^{k} T^{*} E \otimes \hat{T}\right)$, and which gives rise to a closed $\hat{T}$-valued $(k+1)$-form $\omega=-d \theta$ : we propose to call it a polylagrangian form. This term is motivated by the observation that the vertical bundle for the projection $\pi^{k}$ onto $E$ is a distinguished lagrangian (maximal isotropic) subbundle of the tangent bundle of $\bigwedge^{k} T^{*} E \otimes \hat{T}$, and the existence of such a "polylagrangian subbundle" will turn out to play a central role in our general definition of polysymplectic and polylagrangian structures.

In terms of standard local coordinates $\left(q^{i}, p_{i}\right)$ for $T^{*} E$ and a basis $\left\{\hat{e}_{a} \mid 1 \leqslant a \leqslant \hat{n}\right\}$ of $\hat{T}$, we have

$$
\hat{\theta}=p_{i}^{a} d q^{i} \otimes \hat{e}_{a}
$$

SO

$$
\hat{\omega}=d q^{i} \wedge d p_{i}^{a} \otimes \hat{e}_{a},
$$

in the polysymplectic case and

$$
\hat{\theta}=\frac{1}{k !} p_{i_{1} \ldots i_{k}}^{a} d q^{i_{1}} \wedge \ldots \wedge d q^{i_{k}} \otimes \hat{e}_{a}
$$

SO

$$
\hat{\omega}=-\frac{1}{k !} d p_{i_{1} \ldots i_{k}}^{a} \wedge d q^{i_{1}} \wedge \ldots \wedge d q^{i_{k}} \otimes \hat{e}_{a},
$$

in the polylagrangian case. In a more general context, when $\bigwedge^{k} T^{*} E \otimes \hat{T}$ is replaced by a manifold $P$, Darboux's theorem guarantees the existence of canonical local coordinates in which $\hat{\omega}$ is given by the expression in equation (16), under appropriate conditions on the form $\hat{\omega}$. Again, the central question is to figure out what precisely are these conditions.

It should be pointed out that for the polysymplectic case, this problem has been solved in Ref. [15], but the fundamental role of what we call the polylagrangian subbundle is not fully appreciated there. As it turns out, this object and its basic properties are the key to the entire subject, allowing to generalize the proof of Darboux's theorem not only from the polysymplectic to the polylagrangian case but also from polysymplectic/polylagrangian structures on manifolds to polysymplectic/polylagrangian structures on the total spaces of fiber bundles - a concept that conveys a precise mathematical meaning to the idea of 
a "smooth family of polysymplectic/polylagrangian structures" (each fiber is a polysymplectic/polylagrangian manifold in such a way that the entire structure depends smoothly on the points of the base manifold). This extension is particularly useful in that it finally allows to formulate in precise mathematical terms what is the relation between multisymplectic and polysymplectic structures and, more generally, between multilagrangian and polylagrangian structures: the latter appear as the "leading order term" of the former, through a simple formal construction that we introduce below and propose to call the "symbol" because it strongly resembles the construction of the symbol of a differential operator.

\section{Polylagrangian forms on vector spaces}

We begin by briefly recalling a few basic notions involving vector-valued alternating multilinear forms. Given finite-dimensional real vector spaces 1$\}$ and $\hat{T}$, we consider $\hat{T}$-valued $(k+1)$-forms $\hat{\omega}$ on $V$,

$$
\hat{\omega} \in \bigwedge^{k+1} V^{*} \otimes \hat{T}
$$

The contraction of such a form $\hat{\omega}$ is the linear map $\hat{\omega}^{b}: V \rightarrow \bigwedge^{k} V^{*} \otimes \hat{T}$ given by

$$
\hat{\omega}^{b}(v)=\mathrm{i}_{v} \hat{\omega},
$$

and the kernel of $\hat{\omega}$ is defined to be the kernel of $\hat{\omega}^{b}$ : ker $\hat{\omega}=\operatorname{ker} \hat{\omega}^{b}$. If ker $\hat{\omega}=\{0\}$ we say that $\hat{\omega}$ is non-degenerate. Given a linear form $\hat{t}^{*} \in \hat{T}^{*}$ on $\hat{T}$, the projection of $\hat{\omega}$ along $\hat{t}^{*}$ is the ordinary $(k+1)$-form on $V$ given by 2

$$
\omega_{\hat{t}^{*}}=\left\langle\hat{t}^{*}, \hat{\omega}\right\rangle \text {. }
$$

Note that $\omega_{\hat{t}^{*}}$ depends linearly on $\hat{t}^{*}$, so if we choose a basis $\left\{\hat{e}_{1}, \ldots, \hat{e}_{\hat{n}}\right\}$ of $\hat{T}$, with dual basis $\left\{\hat{e}^{1}, \ldots, \hat{e}^{\hat{n}}\right\}$ of $\hat{T}^{*}$, we have

$$
\hat{\omega}=\omega^{a} \otimes \hat{e}_{a} \quad \text { with } \quad \omega^{a}=\omega_{\hat{e}^{a}} \quad(1 \leqslant a \leqslant \hat{n}) .
$$

Then it is clear that

$$
\operatorname{ker} \hat{\omega}=\bigcap_{\hat{t}^{*} \in \hat{T}^{*}} \operatorname{ker} \omega_{\hat{t}^{*}}=\bigcap_{a=1}^{\hat{n}} \operatorname{ker} \omega^{a} .
$$

Next, suppose that $L$ is a subspace of $V$ and $\ell$ is an integer satisfying $1 \leqslant \ell \leqslant k$; then extending the definition given in Ref. [9] from ordinary to vector-valued forms, we define

\footnotetext{
${ }^{1}$ In order to simplify the presentation, we assume all vector spaces involved to be real and finitedimensional: the extension to vector spaces over an arbitrary field of characteristic 0 is straightforward, and generalization to the infinite-dimensional setting, which requires imposing appropriate continuity conditions from functional analysis, will be left to a possible future investigation.

${ }^{2}$ Throughout this paper the symbol $\langle.,$.$\rangle will stand for the natural bilinear pairing between a vector$ space and its dual.
} 
the $\ell$-orthogonal complement of $L$ (with respect to $\hat{\omega}$ ) to be the subspace $L^{\hat{\omega}, \ell}$ of $V$ given by

$$
L^{\hat{\omega}, \ell}=\left\{v \in V \mid \mathrm{i}_{v} \mathrm{i}_{v_{1}} \ldots \mathrm{i}_{v_{\ell}} \hat{\omega}=0 \text { for all } v_{1}, \ldots, v_{\ell} \in L\right\} .
$$

Note that these orthogonal complements form an increasing sequence under inclusion:

$$
L^{\hat{\omega}, 1} \subset \ldots \subset L^{\hat{\omega}, k}
$$

We say that $L$ is $\ell$-isotropic (with respect to $\hat{\omega}$ ) if $L \subset L^{\hat{\omega}, \ell}$ and is maximal $\ell$-isotropic or, more briefly, $\ell$-lagrangian (with respect to $\hat{\omega}$ ) if it is maximal in the partially ordered set formed by the $\ell$-isotropic subspaces of $V$; it is a simple exercise to check that, as usual, this is the case if and only if $L=L^{\hat{\omega}, \ell}$. If $\ell=1$ we omit the prefix 1 and may conclude that a subspace $L$ of $V$ will be isotropic if and only if 4

$$
\hat{\omega}^{b}(L) \subset \hat{\omega}^{b}(V) \cap\left(\bigwedge^{k} L^{\perp}\right) \otimes \hat{T}
$$

and will be maximal isotropic if and only if $\operatorname{ker} \hat{\omega} \subset L$ and

$$
\hat{\omega}^{b}(L)=\hat{\omega}^{b}(V) \cap\left(\bigwedge^{k} L^{\perp}\right) \otimes \hat{T} .
$$

(For explicit proofs of these elementary statements, the reader may consult Ref. [16].) At first sight, the intersection with the subspace $\hat{\omega}^{b}(V)$ on the rhs of these relations may seem strange, in particular since in the inclusion stated in equation (24) it is really superfluous, but that is by no means the case for the equality stated in equation (25). Rather, omitting this intersection leads to a strengthened form of equation (25) which turns out to provide the key to the theory of polysymplectic and, more generally, of polylagrangian forms:

Definition 1 Let $V$ and $\hat{T}$ be finite-dimensional vector spaces, with $\hat{n} \equiv \operatorname{dim} \hat{T}$, and let $\hat{\omega}$ be a non-vanishing $\hat{T}$-valued $(k+1)$-form on $V$. We say that $\hat{\omega}$ is a polylagrangian form of rank $N$ if $V$ admits a subspace $L$ of codimension $N$ which is polylagrangian, i.e., such that

$$
\hat{\omega}^{b}(L)=\left(\bigwedge^{k} L^{\perp}\right) \otimes \hat{T} .
$$

When $k=1$ and $\hat{\omega}$ is non-degenerate, we call $\hat{\omega}$ a polysymplectic form. If the condition of non-degeneracy is dropped, we speak of a polypresymplectic form.

As a first property of polylagrangian forms, we note that a polylagrangian subspace, when it exists, contains the kernel of $\hat{\omega}$ and hence really is a special type of maximal isotropic subspace. But more than that is true.

\footnotetext{
${ }^{3}$ We discard the trivial case $\ell=0$ since extrapolating the definition to this case would lead to the conclusion that $L^{\hat{\omega}, 0}$ is simply the kernel of $\hat{\omega}$, independently of the subspace $L$ of $V$.

${ }^{4}$ Throughout this paper the symbol.${ }^{\perp}$ will denote the annihilator of a subspace, i.e., given a subspace $L$ of a vector space $V, L^{\perp}$ is the subspace of its dual space $V^{*}$ consisting of the linear forms on $V$ that vanish on $L$.
} 
Proposition 1 Let $V$ and $\hat{T}$ be finite-dimensional vector spaces, with $\hat{n} \equiv \operatorname{dim} \hat{T}$, and let $\hat{\omega}$ be a $\hat{T}$-valued polylagrangian $(k+1)$-form on $V$ of rank $N$, with polylagrangian subspace $L$. Then $N \geqslant k$, and $L$ contains the kernel of $\hat{\omega}$ as well as the kernel of each of the projected forms $\omega_{\hat{t}^{*}}\left(\hat{t}^{*} \in \hat{T}^{*} \backslash\{0\}\right)$ :

$$
\text { ker } \hat{\omega} \subset L \quad, \quad \operatorname{ker} \omega_{\hat{t}^{*}} \subset L \quad \text { for all } \hat{t}^{*} \in \hat{T}^{*} \backslash\{0\} \text {. }
$$

Proof: $\quad$ First we observe that if $N<k$, we have $\bigwedge^{k} L^{\perp}=\{0\}$, so both sides of the equation (26) vanish, i.e., $L$ is contained in ker $\hat{\omega}$ and hence ker $\hat{\omega}$ has codimension $<k$ in $V$, implying $\hat{\omega} \equiv 0$, since the $(k+1)$-form on the quotient space $V /$ ker $\hat{\omega}$ induced by $\hat{\omega}$ vanishes identically. (More generally, this argument shows that a nonvanishing vector-valued $(k+1)$-form does not permit isotropic subspaces of codimension $<k$.) Thus supposing that $\operatorname{dim} L^{\perp}=N \geqslant k$, we can for any vector $v \in V \backslash L$ find a linearly independent set of 1-forms $v_{1}^{*}, \ldots, v_{k}^{*} \in L^{\perp}$ such that $\left\langle v_{1}^{*}, v\right\rangle=1$ and $\left\langle v_{i}^{*}, v\right\rangle=0$ for $i>1$. Given $\hat{t}^{*} \in \hat{T}^{*}$, take $\hat{t} \in \hat{T}$ such that $\left\langle\hat{t}^{*}, \hat{t}\right\rangle=1$. According to the definition of a polylagrangian subspace, there is a vector $u \in L$ such that

$$
\mathrm{i}_{u} \hat{\omega}=v_{1}^{*} \wedge \ldots \wedge v_{k}^{*} \otimes \hat{t} \quad \Rightarrow \quad \mathrm{i}_{v} \mathrm{i}_{u} \omega_{\hat{t}^{*}}=v_{2}^{*} \wedge \ldots \wedge v_{k}^{*} \neq 0
$$

and so $v \notin \operatorname{ker} \omega_{\hat{t}^{*}}$. Hence it follows that $\operatorname{ker} \hat{\omega} \subset \operatorname{ker} \omega_{\hat{t}^{*}} \subset L$.

On the other hand, considering the case of main interest, which is that of 2 -forms, it must be emphasized that, as shown by the counterexamples presented in Appendix A, by far not every vector-valued 2 -form is poly(pre)symplectic, which means that in contrast to lagrangian subspaces, a polylagrangian subspace need not exist, and even if it does exist, not every lagrangian subspace is polylagrangian. However, there is a simple dimension criterion that allows to decide whether a given isotropic subspace is polylagrangian:

Proposition 2 Let $V$ and $\hat{T}$ be finite-dimensional vector spaces, with $\hat{n} \equiv \operatorname{dim} \hat{T}$, and let $\hat{\omega}$ be a non-vanishing $\hat{T}$-valued $(k+1)$-form on $V$. Given any subspace $L$ of $V$, with $N=\operatorname{dim}(V / L)$, such that $N \geqslant k$, the following statements are equivalent:

- $L$ is a polylagrangian subspace and $\hat{\omega}$ is a polylagrangian form of rank $N$.

- L contains ker $\hat{\omega}$, is isotropic and has dimension

$$
\operatorname{dim} L=\operatorname{dim} \operatorname{ker} \hat{\omega}+\hat{n}\left(\begin{array}{l}
N \\
k
\end{array}\right) .
$$

Proof: $\quad$ Taking into account that, for any isotropic subspace $L$ of $V$ containing the kernel of $\hat{\omega}$, the contraction map $\hat{\omega}^{b}$ induces an injective linear map of $L /$ ker $\hat{\omega}$ into $\left(\bigwedge^{k} L^{\perp}\right) \otimes \hat{T}$, the result follows from an elementary dimension count.

The case of non-degenerate ordinary or scalar polylagrangian forms $(\operatorname{dim} \hat{T}=1)$ has been studied in the literature [8-10] under the label "multisymplectic forms" (a terminology 
that we propose to abandon since we use this term in a different sense; see Definition 3 below), but the concept of polylagrangian subspace appears only implicitly, namely through the dimension criterion formulated in Proposition 2 above, which is employed as a definition, so that it remains unclear how to extend this purely numerical recipe to other situations, in particular when $\hat{\omega}$ is taken to be a truly vector-valued form $(\operatorname{dim} \hat{T}>1)$. The main statement here is

Proposition 3 Let $V$ be a finite-dimensional vector space and let $\hat{\omega}$ be a scalar polylagrangian $(k+1)$-form on $V$ of rank $N$, with polylagrangian subspace $L$. Then any isotropic subspace $\tilde{L}$ containing the kernel of $\hat{\omega}$ and such that

$$
\operatorname{dim} \tilde{L}>\operatorname{dim} \operatorname{ker} \hat{\omega}+\left(\begin{array}{c}
N-1 \\
k
\end{array}\right)+1
$$

is contained in $L$. In particular, if $N>k>1, L$ is unique.

Remark 1 Note that the uniqueness statement for $L$ is of course false for symplectic forms $(k=1)$ and also for volume forms $(N=k)$ : in both cases, isotropic subspaces $\tilde{L}$ satisfying the dimension condition (29) do not exist, and there is no restriction whatsoever on the relative position of lagrangian subspaces (which for a symplectic form on a $(2 N)$ dimensional space are $N$-dimensional and for a volume form are one-dimensional).

Proof: Obviously, passing from $V$ to the quotient space $V /$ ker $\hat{\omega}$ if necessary, and taking into account the previous remark, we may assume without loss of generality that $\hat{\omega}$ is non-degenerate and also that $N>k>1$. Following Refs $[8,10]$, we begin by showing that any isotropic subspace of $V$ of dimension greater than 1 must intersect $L$ non-trivially. Indeed, if $v_{1}$ and $v_{2}$ are linearly independent vectors in $V$ such that the two-dimensional subspace $\operatorname{span}\left(v_{1}, v_{2}\right)$ generated by $v_{1}$ and $v_{2}$ satisfies $\operatorname{span}\left(v_{1}, v_{2}\right) \cap L=\{0\}$, we can find a basis $\left\{e_{1}, \ldots, e_{N}\right\}$ of a subspace of $V$ complementary to $L$ such that $e_{1}=v_{1}$ and $e_{2}=v_{2}$; then denoting the corresponding dual basis of $L^{\perp}$ by $\left\{e^{1}, \ldots, e^{N}\right\}$, we use the fact that $L$ is polylagrangian to conclude that there exists a vector $u$ in $L$ such that $\mathrm{i}_{u} \hat{\omega}=e^{1} \wedge \ldots \wedge e^{k}$, so $\hat{\omega}\left(u, e_{1}, \ldots, e_{k}\right)=1$ and hence $\mathrm{i}_{v_{1}} \mathrm{i}_{v_{2}} \hat{\omega}$ cannot vanish, i.e., $\operatorname{span}\left(v_{1}, v_{2}\right)$ cannot be isotropic. Using this result, we conclude that if $\tilde{L}$ is any isotropic subspace of $V$, then the codimension of $\tilde{L} \cap L$ in $\tilde{L}$ is at most 1 , so

$$
\operatorname{dim}(\tilde{L}+L)-\operatorname{dim} L=\operatorname{dim} \tilde{L}-\operatorname{dim}(\tilde{L} \cap L) \leqslant 1
$$

Suppose now that $\tilde{L}$ is an isotropic subspace of $V$ such that

$$
\operatorname{dim}(\tilde{L}+L)-\operatorname{dim} L=\operatorname{dim} \tilde{L}-\operatorname{dim}(\tilde{L} \cap L)=1 .
$$

Then $\tilde{L}+L$ has codimension $N-1$ in $V$ and hence

$$
\operatorname{dim} \bigwedge^{k}(\tilde{L}+L)^{\perp}=\left(\begin{array}{c}
N-1 \\
k
\end{array}\right)
$$


whereas, by hypothesis,

$$
\operatorname{dim}(\tilde{L} \cap L)>\left(\begin{array}{c}
N-1 \\
k
\end{array}\right)
$$

which contradicts the fact that the restriction of the linear isomorphism $\left.\hat{\omega}^{b}\right|_{L}: L \rightarrow \bigwedge^{k} L^{\perp}$ maps the subspace $\tilde{L} \cap L$ of $L$ injectively into the subspace $\bigwedge^{k}(\tilde{L}+L)^{\perp}$ of $\bigwedge^{k} L^{\perp}$.

What is remarkable is that the above uniqueness statement remains valid for vector-valued polylagrangian forms $(\operatorname{dim} \hat{T}>1)$ and that in this case it becomes true even when $k=1$ or $N=k$ : this is a consequence of the following explicit construction of $L$.

Theorem 1 Let $V$ and $\hat{T}$ be finite-dimensional vector spaces, with $\hat{n} \equiv \operatorname{dim} \hat{T} \geqslant 2$, and let $\hat{\omega}$ be a $\hat{T}$-valued polylagrangian $(k+1)$-form of rank $N$ on $V$, with polylagrangian subspace $L$. Then $L$ is given by

$$
L=\sum_{t^{*} \in \hat{T}^{*} \backslash\{0\}} \operatorname{ker} \omega_{\hat{t}^{*}}
$$

and, in particular, is unique. In terms of a basis $\left\{\hat{e}_{1}, \ldots, \hat{e}_{\hat{n}}\right\}$ of $\hat{T}$ with dual basis $\left\{\hat{e}^{1}, \ldots, \hat{e}^{\hat{n}}\right\}$ of $\hat{T}^{*}$,

$$
L=\operatorname{ker} \hat{\omega} \oplus K_{1} \oplus \ldots \oplus K_{\hat{n}}
$$

and for $1 \leqslant a \leqslant \hat{n}$,

$$
L=\operatorname{ker} \omega^{a} \oplus K_{a},
$$

where for $1 \leqslant a \leqslant \hat{n}, K_{a}$ is a subspace of $V$ chosen so that

$$
\bigcap_{\substack{b=1 \\ b \neq a}}^{\hat{n}} \operatorname{ker} \omega^{b}=\operatorname{ker} \hat{\omega} \oplus K_{a} .
$$

The dimensions of these various subspaces are given by

$$
\operatorname{dim} \operatorname{ker} \omega^{a}=\operatorname{dim} \operatorname{ker} \hat{\omega}+(\hat{n}-1)\left(\begin{array}{c}
N \\
k
\end{array}\right), \quad \operatorname{dim} K_{a}=\left(\begin{array}{c}
N \\
k
\end{array}\right) .
$$

Proof: $\quad$ Fix a basis $\left\{\hat{e}_{1}, \ldots, \hat{e}_{\hat{n}}\right\}$ of $\hat{T}$ with dual basis $\left\{\hat{e}^{1}, \ldots, \hat{e}^{\hat{n}}\right\}$ of $\hat{T}^{*}$ and choose subspaces $K_{a}$ of $V(a=1, \ldots, \hat{n})$ as indicated above. Then the subspaces ker $\hat{\omega}$ and $K_{1}, \ldots, K_{\hat{n}}$ of $V$ have trivial intersection, so their sum is direct and defines a subspace of $V$ which we shall, for the moment, denote by $L^{\prime}$. According to the previous proposition, $L^{\prime} \subset L$. To show that $L^{\prime}=L$, it is therefore sufficient to prove that $\hat{\omega}^{b}(L) \subset \hat{\omega}^{b}\left(L^{\prime}\right)$, since both $L$ and $L^{\prime}$ contain ker $\hat{\omega}$. Using the definition of a polylagrangian subspace, we conclude that we must establish the inclusion

$$
\left(\bigwedge^{k} L^{\perp}\right) \otimes \hat{T} \subset \hat{\omega}^{b}\left(L^{\prime}\right)
$$


But the equality (26) guarantees that for any $\alpha \in \bigwedge^{k} L^{\perp}$ and for $1 \leqslant a \leqslant \hat{n}$, there is a vector $v_{a} \in L$ such that

$$
\mathrm{i}_{v_{a}} \hat{\omega}=\alpha \otimes \hat{e}_{a} .
$$

Then

$$
\mathrm{i}_{v_{a}} \hat{\omega}^{b}=\alpha\left\langle\hat{e}^{b}, \hat{e}_{a}\right\rangle=\delta_{a}^{b} \alpha
$$

so we see that

$$
v_{a} \in \bigcap_{\substack{b=1 \\ b \neq a}}^{\hat{n}} \operatorname{ker} \hat{\omega}^{b} .
$$

Decomposing $v_{a}$ according to equation (33), we find a vector $u_{a} \in K_{a}$ such that

$$
\mathrm{i}_{u_{a}} \hat{\omega}=\alpha \otimes \hat{e}_{a}
$$

so $\alpha \otimes \hat{e}_{a} \in \hat{\omega}^{b}\left(K_{a}\right) \subset \hat{\omega}^{b}\left(L^{\prime}\right)$. Conversely, if $u_{a} \in K_{a} \subset L$, then $\mathrm{i}_{u_{a}} \hat{\omega}^{b}=0$ for $b \neq a$ and hence $i_{u_{a}} \hat{\omega}$ is of the form $\alpha \otimes \hat{e}_{a}$ for some $\alpha \in \Lambda^{k} L^{\perp}$. Thus we conclude that $\hat{\omega}^{b}$ maps $K_{a}$ isomorphically onto $\left(\bigwedge^{k} L^{\perp}\right) \otimes \operatorname{span}\left(\hat{e}_{a}\right)$, where $\operatorname{span}\left(\hat{e}_{a}\right)$ denotes the one-dimensional subspace of $\hat{T}$ generated by $\hat{e}_{a}$, which proves the second formula in equation (34). Finally, we observe that combining equations (21) and (33) gives

$$
\operatorname{ker} \omega^{a} \cap K_{a}=\{0\}
$$

so that equation (32) follows from equation (31), whereas the first formula in equation (34) is now a direct consequence of equations (28) and (32).

Another fundamental property of polylagrangian forms is that the polylagrangian subspace has a particular type of direct complement.

Theorem 2 Let $V$ and $\hat{T}$ be finite-dimensional vector spaces and let $\hat{\omega}$ be a $\hat{T}$-valued polylagrangian $(k+1)$-form of rank $N$ on $V$, with polylagrangian subspace $L$. Then there exists a $k$-isotropic subspace $E$ of $V$ complementary to $L$, i.e., such that

$$
V=E \oplus L
$$

Proof: Let $E_{0}$ be a $k$-isotropic subspace of $V$ of dimension $N^{\prime}$ such that $E_{0} \cap L=\{0\}$. (For instance, as long as $N^{\prime} \leqslant k, E_{0}$ can be any subspace of $V$ such that $E_{0} \cap L=\{0\}$.) If $N^{\prime}=N$, we are done. Otherwise, choose a basis $\left\{e_{1}, \ldots, e_{N}\right\}$ of a subspace of $V$ complementary to $L$ such that the first $N^{\prime}$ vectors constitute a basis of $E_{0}$, and denote the corresponding dual basis of $L^{\perp}$ by $\left\{e^{1}, \ldots, e^{N}\right\}$. We shall prove that there exists a vector $u \in V \backslash\left(E_{0} \oplus L\right)$ such that the subspace $E_{1}$ of $V$ spanned by $u$ and $E_{0}$ is $k$-isotropic and satisfies $E_{1} \cap L=\{0\}$; then since $\operatorname{dim} E_{1}=N^{\prime}+1$, the statement of the theorem follows by induction. To this end, consider an arbitrary basis $\left\{\hat{e}_{a} \mid 1 \leqslant a \leqslant \hat{n}\right\}$ of $\hat{T}$ with dual basis $\left\{\hat{e}^{a} \mid 1 \leqslant a \leqslant \hat{n}\right\}$ of $\hat{T}^{*}$ and, choosing any subspace $L^{\prime}$ of $L$ complementary to 
ker $\hat{\omega}$, use the fact that $L$ is polylagrangian to conclude that there exists a unique basis $\left\{e_{a}^{i_{1} \ldots i_{k}} \mid 1 \leqslant a \leqslant \hat{n}, 1 \leqslant i_{1}<\ldots<i_{k} \leqslant N\right\}$ of $L^{\prime}$ such that

$$
\hat{\omega}^{b}\left(e_{a}^{i_{1} \ldots i_{k}}\right)=e^{i_{1}} \wedge \ldots \wedge e^{i_{k}} \otimes \hat{e}_{a} .
$$

Thus, for $1 \leqslant i_{1}<\ldots<i_{k} \leqslant N$ and $1 \leqslant j_{1}<\ldots<j_{k} \leqslant N$, we have

$$
\omega^{b}\left(e_{a}^{i_{1} \ldots i_{k}}, e_{j_{1}}, \ldots, e_{j_{k}}\right)=\delta_{a}^{b} \delta_{j_{1}}^{i_{1}} \ldots \delta_{j_{k}}^{i_{k}}
$$

Therefore, the vector

$$
u=e_{N^{\prime}+1}-\frac{1}{k !} \omega^{a}\left(e_{N^{\prime}+1}, e_{i_{1}}, \ldots, e_{i_{k}}\right) e_{a}^{i_{1} \ldots i_{k}}
$$

does not belong to the subspace $E_{0} \oplus L$ and, for $1 \leqslant j_{1}<\ldots<j_{k} \leqslant N$, satisfies

$$
\begin{aligned}
& \omega^{b}\left(u, e_{j_{1}}, \ldots, e_{j_{k}}\right) \\
& \quad=\omega^{b}\left(e_{N^{\prime}+1}, e_{j_{1}}, \ldots, e_{j_{k}}\right)-\frac{1}{k !} \omega^{a}\left(e_{N^{\prime}+1}, e_{i_{1}}, \ldots, e_{i_{k}}\right) \omega^{b}\left(e_{a}^{i_{1} \ldots i_{k}}, e_{j_{1}}, \ldots, e_{j_{k}}\right) \\
& \quad=0
\end{aligned}
$$

which implies that since the subspace $E_{0}$ spanned by $e_{1}, \ldots, e_{N^{\prime}}$ is $k$-isotropic, the subspace $E_{1}$ spanned by $e_{1}, \ldots, e_{N^{\prime}}$ and $u$ is so as well.

Example 1 (The canonical form) Let $E$ and $\hat{T}$ be vector spaces of dimension $N$ and $\hat{n}$, respectively. Set

$$
V_{0}=E \oplus\left(\left(\bigwedge^{k} E^{*}\right) \otimes \hat{T}\right)
$$

The canonical polylagrangian form of rank $N$ is the non-degenerate $\hat{T}$-valued $(k+1)$ form $\hat{\omega}_{0}$ on $V_{0}$ defined by

$$
\hat{\omega}_{0}\left(\left(u_{0}, \alpha_{0} \otimes \hat{t}_{0}\right), \ldots,\left(u_{k}, \alpha_{k} \otimes \hat{t}_{k}\right)\right)=\sum_{i=0}^{k}(-1)^{i} \alpha_{i}\left(u_{0}, \ldots, \widehat{u}_{i}, \ldots, u_{k}\right) \hat{t}_{i} .
$$

If $k=1$ we shall call $\hat{\omega}_{0}$ the canonical polysymplectic form.

For scalar forms $(\hat{T}=\mathbb{R})$ this construction can be found, e.g., in Refs [8-10].

To justify this terminology, note that it is a straightforward exercise to show that $\hat{\omega}_{0}$ is non-degenerate and that, considering $E$ and

$$
L=\left(\bigwedge^{k} E^{*}\right) \otimes \hat{T}
$$

as subspaces of $V_{0}$, we have the direct decomposition $V_{0}=E \oplus L$ where

$L$ is polylagrangian and $E$ is $k$-isotropic . 
In terms of bases, let $\left\{\hat{e}_{a} \mid 1 \leqslant a \leqslant \hat{n}\right\}$ be any basis of $\hat{T}$ with dual basis $\left\{\hat{e}^{a} \mid 1 \leqslant a \leqslant \hat{n}\right\}$ of $\hat{T}^{*}$ and let $\left\{e_{i} \mid 1 \leqslant i \leqslant N\right\}$ be any basis of $E$ with dual basis $\left\{e^{i} \mid 1 \leqslant i \leqslant N\right\}$ of $E^{*}$. For $1 \leqslant a \leqslant \hat{n}$ and $1 \leqslant i_{1}<\ldots<i_{k} \leqslant N$, define

$$
e_{a}^{i_{1} \ldots i_{k}}=e^{i_{1}} \wedge \ldots \wedge e^{i_{k}} \otimes \hat{e}_{a} \quad, \quad e_{i_{1} \ldots i_{k}}^{a}=e_{i_{1}} \wedge \ldots \wedge e_{i_{k}} \otimes \hat{e}^{a} .
$$

This provides a basis $\left\{e_{i}, e_{a}^{i_{1} \ldots i_{k}} \mid 1 \leqslant a \leqslant \hat{n}, 1 \leqslant i \leqslant N, 1 \leqslant i_{1}<\ldots<i_{k} \leqslant N\right\}$ of $V_{0}$ with dual basis $\left\{e^{i}, e_{i_{1} \ldots i_{k}}^{a} \mid 1 \leqslant a \leqslant \hat{n}, 1 \leqslant i \leqslant N, 1 \leqslant i_{1}<\ldots<i_{k} \leqslant N\right\}$ of $V_{0}^{*}$, both of which we shall refer to as a canonical basis or Darboux basis, such that

$$
\hat{\omega}_{0}=\frac{1}{k !}\left(e_{i_{1} \ldots i_{k}}^{a} \wedge e^{i_{1}} \wedge \ldots \wedge e^{i_{k}}\right) \otimes \hat{e}_{a}
$$

Now it is easy to derive the algebraic Darboux theorem for general polylagrangian forms: let $\left\{\hat{e}_{a} \mid 1 \leqslant a \leqslant \hat{n}\right\}$ be an arbitrary basis of $\hat{T}$, with dual basis $\left\{\hat{e}^{a} \mid 1 \leqslant a \leqslant \hat{n}\right\}$ of $\hat{T}^{*}$, and let $\left\{e_{i} \mid 1 \leqslant i \leqslant N\right\}$ be an arbitrary basis of a $k$-isotropic subspace $E$ complementary to $L$ in $V$, with dual basis $\left\{e^{i} \mid 1 \leqslant i \leqslant N\right\}$ of $L^{\perp} \cong E^{*}$. Choosing an arbitrary subspace $L^{\prime}$ of $L$ complementary to ker $\hat{\omega}$ and taking into account the identity (26), we define a basis $\left\{e_{a}^{i_{1} \ldots i_{k}} \mid 1 \leqslant a \leqslant \hat{n}, 1 \leqslant i_{1}<\ldots<i_{k} \leqslant N\right\}$ of $L^{\prime}$ by

$$
\hat{\omega}^{b}\left(e_{a}^{i_{1} \ldots i_{k}}\right)=e^{i_{1}} \wedge \ldots \wedge e^{i_{k}} \otimes \hat{e}_{a} .
$$

It is easy to see that the union of this basis with that of $E$ gives a canonical basis of $V$ (or more precisely, of $E \oplus L^{\prime}$, which is a subspace of $V$ complementary to ker $\hat{\omega}$ ). Thus we have proved 5

\section{Theorem 3 (Darboux theorem for polylagrangian vector spaces)}

Every polylagrangian vector space admits a canonical basis.

\section{Multilagrangian forms on vector spaces}

In this section we deal with ordinary alternating multilinear forms which are partially horizontal with respect to a given vertical subspace. To explain what this means, assume that we are given a finite-dimensional real vector space $W$ together with a fixed subspace $V$ and a projection $\pi$ from $W$ to another finite-dimensional real vector space $T$ which has $V$ as its kernel, so that $T \cong W / V$, i.e., we have a short exact sequence of vector spaces 1

$$
0 \longrightarrow V \longrightarrow W \stackrel{\pi}{\longrightarrow} T \longrightarrow 0
$$

Motivated by standard jargon of fiber bundle theory, we shall refer to $W$ as the total space, $V$ as the vertical space and $T$ as the base space. Then an $r$-form $\alpha \in \bigwedge^{r} W^{*}$

\footnotetext{
${ }^{5}$ Clearly, the inductive construction of a $k$-isotropic subspace $E$ complementary to the polylagrangian subspace $L$, as presented in the proof of Theorem 2, provides an explicit iteration procedure for building polylagrangian bases in a way similar to the well known Gram-Schmidt orthogonalization process.
} 
on $W$ is said to be $(r-s$ )-horizontal (with respect to $\pi$ ), where $0 \leqslant s \leqslant r$, if its contraction with more than $s$ vertical vectors vanishes, i.e., if

$$
\mathrm{i}_{v_{1}} \ldots \mathrm{i}_{v_{s+1}} \alpha=0 \quad \text { for } v_{1}, \ldots, v_{s+1} \in V .
$$

The vector space of $(r-s)$-horizontal $r$-forms on $W$ will be denoted by $\bigwedge_{s}^{r} W^{*}$. Note that as $s$ is varied (with $r$ fixed), these spaces form an increasing sequence under inclusion:6

$$
\bigwedge^{r} T^{*} \cong \bigwedge_{0}^{r} W^{*} \subset \ldots \subset \bigwedge_{s}^{r} W^{*} \subset \ldots \subset \bigwedge_{r}^{r} W^{*}=\bigwedge^{r} W^{*} .
$$

At the two extremes, we have $\bigwedge_{r}^{r} W^{*}=\bigwedge^{r} W^{*}$ since the condition of 0-horizontality is void, whereas the space $\bigwedge_{0}^{r} W^{*}$ of fully horizontal $r$-forms on $W$, which are precisely the horizontal $r$-forms as defined in [17, Vol. 2] and [18, Vol. 1], is canonically isomorphic to the space $\bigwedge^{r} T^{*}$ of all $r$-forms on $T: \bigwedge_{0}^{r} W^{*} \cong \bigwedge^{r} T^{*}$. This canonical isomorphism is simply given by pull-back with the projection $\pi$, i.e., $\alpha_{W}=\pi^{*} \alpha_{T}$ or

$$
\alpha_{W}\left(w_{1}, \ldots, w_{r}\right)=\alpha_{T}\left(\pi\left(w_{1}\right), \ldots, \pi\left(w_{r}\right)\right) \quad \text { for } w_{1}, \ldots, w_{r} \in W .
$$

Its inverse is given by $\alpha_{T}=\mathfrak{s}^{*} \alpha_{W}$ or

$$
\alpha_{T}\left(t_{1}, \ldots, t_{r}\right)=\alpha_{W}\left(\mathfrak{s}\left(t_{1}\right), \ldots, \mathfrak{s}\left(t_{r}\right)\right) \quad \text { for } t_{1}, \ldots, t_{r} \in T,
$$

where $\mathfrak{s}$ is any splitting of the exact sequence (41), i.e., any linear mapping from $T$ to $W$ such that $\pi \circ \mathfrak{s}=\mathrm{id}_{T} 7$ Extending this construction to partially horizontal forms leads us naturally to the concept of symbol, which will provide the link between polysymplectic/ polylagrangian and multisymplectic/multilagrangian structures.

Definition 2 Let $W, V$ and $T$ be finite-dimensional vector spaces related by the short exact sequence (41). The symbol of an $(r-s)$-horizontal $r$-form $\alpha$ on $W, \alpha \in \bigwedge_{s}^{r} W^{*}$, is the $\bigwedge^{r-s} T^{*}$-valued s-form $\hat{\alpha}$ on $V, \hat{\alpha} \in \bigwedge^{s} V^{*} \otimes \bigwedge^{r-s} T^{*}$, given by

$$
\hat{\alpha}\left(v_{1}, \ldots, v_{s}\right)=\mathrm{i}_{v_{1}} \ldots \mathrm{i}_{v_{s}} \alpha \quad \text { for } v_{1}, \ldots, v_{s} \in V \text {. }
$$

Explicitly, we may use equation (45) to arrive at the following formula for $\hat{\alpha}$ in terms of $\alpha$ :

$$
\begin{gathered}
\hat{\alpha}\left(v_{1}, \ldots, v_{s}\right)\left(t_{1}, \ldots, t_{r-s}\right)=\alpha\left(v_{1}, \ldots, v_{s}, \mathfrak{s}\left(t_{1}\right), \ldots, \mathfrak{s}\left(t_{r-s}\right)\right) \\
\text { for } v_{1}, \ldots, v_{s} \in V, t_{1}, \ldots, t_{r-s} \in T
\end{gathered}
$$

where, once again, $\mathfrak{s}$ is any splitting of the exact sequence (41).7 It follows that passage to the symbol can be regarded as a projection, from the space $\bigwedge_{s}^{r} W^{*}$ of $(r-s)$-horizontal $r$-forms on $W$ to the space $\bigwedge^{s} V^{*} \otimes \bigwedge^{r-s} T^{*}$ of $s$-forms on $V$ with values in the space

\footnotetext{
${ }^{6}$ The first few terms of this sequence may be trivial, since $\bigwedge_{s}^{r} W^{*}=\{0\}$ if $s<r-\operatorname{dim} T$.

${ }^{7}$ It is a straightfoward exercise to verify that the expressions on the rhs of equation (45) and on the rhs of equation (47) do not depend on the choice of the splitting $\mathfrak{s}$ when $\alpha_{W}$ is horizontal and $\alpha$ is $(r-s)$-horizontal.
} 
$\bigwedge^{r-s} T^{*}$ of $(r-s)$-forms on $T$, whose kernel is the subspace $\bigwedge_{s-1}^{r} W^{*}$ of $(r-s+1)$-horizontal $r$-forms on $W$.

If we introduce a basis $\left\{e_{1}^{V}, \ldots, e_{m}^{V}, e_{1}^{T}, \ldots, e_{n}^{T}\right\}$ of $W$ such that the first $m$ vectors span $V$ while the last $n$ vectors span a subspace complementary to $V$ and hence isomorphic to $T$, then in terms of the dual basis $\left\{e_{V}^{1}, \ldots, e_{V}^{m}, e_{T}^{1}, \ldots, e_{T}^{n}\right\}$ of $W^{*}$, an arbitrary form $\alpha \in \bigwedge_{s}^{r} W^{*}$ is represented as 8

$$
\alpha=\sum_{t=0}^{s} \frac{1}{t !} \frac{1}{(r-t) !} \omega_{i_{1} \ldots i_{t} ; \mu_{1} \ldots \mu_{r-t}} e_{V}^{i_{1}} \wedge \ldots \wedge e_{V}^{i_{t}} \wedge e_{T}^{\mu_{1}} \wedge \ldots \wedge e_{T}^{\mu_{r-t}}
$$

while its symbol $\hat{\alpha} \in \bigwedge^{s} V^{*} \otimes \bigwedge^{r-s} T^{*}$ is represented as

$$
\hat{\alpha}=\frac{1}{s !} \frac{1}{(r-s) !} \alpha_{i_{1} \ldots i_{s} ; \mu_{1} \ldots \mu_{r-s}} e_{V}^{i_{1}} \wedge \ldots \wedge e_{V}^{i_{s}} \otimes e_{T}^{\mu_{1}} \wedge \ldots \wedge e_{T}^{\mu_{r-s}}
$$

which also shows that

$$
\operatorname{dim} \bigwedge_{s}^{r} W^{*}=\sum_{t=0}^{s}\left(\begin{array}{c}
\operatorname{dim} V \\
t
\end{array}\right)\left(\begin{array}{c}
\operatorname{dim} T \\
r-t
\end{array}\right)
$$

where it is to be understood that $\left(\begin{array}{l}k \\ l\end{array}\right)=0$ if $l>k$.

As in the polysymplectic and, more generally, the polylagrangian case, our definition of a multisymplectic and, more generally, of a multilagrangian form will be based on the existence of a special type of maximal isotropic subspace, the only restriction being that we consider only isotropic subspaces of the vertical space $V$; correspondingly, the concept of maximality should in this context be understood to mean maximality in the partially ordered set formed by the isotropic subspaces of $V$ (not $W$ ). More precisely, suppose we are given a fixed $(k+1-r)$-horizontal $(k+1)$-form $\omega$ on $W$,

$$
\omega \in \bigwedge_{r}^{k+1} W^{*}
$$

where $1 \leqslant r \leqslant k+1,9$ and denote by $\omega^{b}$ the contraction of $\omega$, as defined in the previous section, which is a linear map $\omega^{b}: W \rightarrow \bigwedge^{k} W^{*}$, as well as its restriction to the vertical subspace $V$, which is a linear map $\omega^{b}: V \rightarrow \bigwedge_{r-1}{ }^{k} W^{*}$. Then defining, for any subspace $L$ of $V$,

$$
\bigwedge_{r-1}^{k} L^{\perp}=\bigwedge^{k} L^{\perp} \cap \bigwedge_{r-1}^{k} W^{*},
$$

${ }^{8}$ The expansion in equation (48) explains why forms in $\bigwedge_{s}^{r} W^{*}$ are called $(r-s)$-horizontal: they are represented as linear combinations of exterior products of 1-forms such that, in each term of the sum, at least $r-s$ of them are horizontal.

${ }^{9}$ The extreme case of fully horizontal forms $(r=0)$ will be excluded right from the start since it can be reduced to the other extreme case where the horizontality condition is void $(r=k+1)$, substituting the total space $W$ by the quotient space $T$. Additional restrictions that serve to exclude other trivial or uninteresting cases will be imposed as we go along. 
we conclude as in the previous section that a subspace $L$ of $V$ will be isotropic (with respect to $\omega$ ) if and only if

$$
\omega^{b}(L) \subset \omega^{b}(V) \cap \bigwedge_{r-1}^{k} L^{\perp}
$$

and will be maximal isotropic (with respect to $\omega$ ) if and only if $V \cap \operatorname{ker} \omega \subset L$ and

$$
\omega^{b}(L)=\omega^{b}(V) \cap \bigwedge_{r-1}^{k} L^{\perp} .
$$

As in the previous section, the intersection with the subspace $\omega^{b}(V)$ on the rhs of these relations is superfluous in equation (53) but not in equation (54), and omitting it here leads to a strengthened form of equation (54) which turns out to provide the key to the theory of multisymplectic and, more generally, of multilagrangian forms:

Definition 3 Let $W, V$ and $T$ be finite-dimensional vector spaces related by the short exact sequence (41), with $n=\operatorname{dim} T$, and let $\omega$ be a non-vanishing $(k+1-r)$-horizontal $(k+1)$-form on $W$, where $1 \leqslant r \leqslant k+1$. We say that $\omega$ is a multilagrangian form of rank $N$ and horizontality degree $k+1-r$ if $V$ admits a subspace $L$ of codimension $N$ which is multilagrangian, i.e., such that

$$
\omega^{b}(L)=\bigwedge_{r-1}^{k} L^{\perp}
$$

When $k=n, r=2$ and $\omega$ is non-degenerate, we call $\omega$ a multisymplectic form. If the condition of non-degeneracy is dropped, we speak of a multipresymplectic form.

The first two propositions on multilagrangian forms are entirely analogous to the corresponding ones for polylagrangian forms. To begin with, we note that a multilagrangian subspace, when it exists, contains the kernel of $\omega$ (implying, in particular, that ker $\omega \subset V$ ) and hence really is a special type of maximal isotropic subspace.

Proposition 4 Let $W, V$ and $T$ be finite-dimensional vector spaces related by the short exact sequence (41), with $n=\operatorname{dim} T$, and let $\omega$ be a multilagrangian $(k+1)$-form on $W$ of rank $N$ and horizontality degree $k+1-r$, where $1 \leqslant r \leqslant k+1$, and with multilagrangian subspace $L$. Then $N+n \geqslant k$ and $k+1-r \leqslant n$, and $L$ contains the kernel of $\omega$ :

$$
\operatorname{ker} \omega \subset L \text {. }
$$

Proof: $\quad$ First we observe that if $N+n<k$, we have $\bigwedge^{k} L^{\perp}=\{0\}$ while if $k+1-r>n$, we have $\bigwedge_{r-1}^{k} W^{*}=\{0\}$, so in either case, both sides of the equation (55) vanish, i.e., $L$ is contained in ker $\omega$ and hence ker $\omega$ has codimension $<k$ in $W$, implying $\omega \equiv 0$, since the $(k+1)$-form on the quotient space $W /$ ker $\omega$ induced by $\omega$ vanishes identically. Thus supposing that $\operatorname{dim} L^{\perp}=N+n \geqslant k$ and using that $\operatorname{dim} V^{\perp}=n$ and $V^{\perp} \subset L^{\perp}$, we conclude that we can, for any vector $w \in W \backslash L$, find a linearly independent set of 1 forms $w_{1}^{*}, \ldots, w_{k}^{*} \in L^{\perp}$ such that $w_{r}^{*}, \ldots, w_{k}^{*} \in V^{\perp},\left\langle w_{1}^{*}, w\right\rangle=1$ and $\left\langle w_{i}^{*}, w\right\rangle=0$ for $i>1$. 
According to the definition of a multilagrangian subspace, there is a vector $u \in L$ such that

$$
\mathrm{i}_{u} \omega=w_{1}^{*} \wedge \ldots \wedge w_{k}^{*} \Rightarrow \mathrm{i}_{w} \mathrm{i}_{u} \omega=w_{2}^{*} \wedge \ldots \wedge w_{k}^{*} \neq 0
$$

and so $w \notin$ ker $\omega$. Hence it follows that $\operatorname{ker} \omega \subset L$.

The second gives a simple dimension criterion that allows to decide whether a given isotropic subspace of $V$ is multilagrangian:

Proposition 5 Let $W, V$ and $T$ be finite-dimensional vector spaces related by the short exact sequence (41), with $n=\operatorname{dim} T$, and let $\omega$ be a non-vanishing $(k+1-r)$-horizontal $(k+1)$-form on $W$, where $1 \leqslant r \leqslant k+1$ and $k+1-r \leqslant n$. Given any subspace $L$ of $V$, with $N=\operatorname{dim}(V / L)$, such that $N+n \geqslant k$, the following statements are equivalent:

- L is a multilagrangian subspace and $\omega$ is a multilagrangian form of rank $N$.

- L contains $\operatorname{ker} \omega$, is isotropic and has dimension

$$
\operatorname{dim} L=\operatorname{dim} \operatorname{ker} \omega+\sum_{s=0}^{r-1}\left(\begin{array}{c}
N \\
s
\end{array}\right)\left(\begin{array}{c}
n \\
k-s
\end{array}\right),
$$

where it is to be understood that $\left(\begin{array}{c}N \\ s\end{array}\right)=0$ if $s>N$.

Proof: $\quad$ Taking into account that, for any isotropic subspace $L$ of $V$ containing the kernel of $\omega$, the contraction map $\omega^{b}$ induces an injective linear map of $L /$ ker $\omega$ into $\bigwedge_{r-1}^{k} L^{\perp}$, we obtain

$$
\operatorname{dim} L-\operatorname{dim} \operatorname{ker} \omega=\operatorname{dim} \omega^{b}(L)=\operatorname{dim} \bigwedge_{r-1}^{k} L^{\perp} .
$$

To calculate this dimension, we introduce a basis $\left\{e_{1}^{L}, \ldots, e_{l}^{L}, e_{1}^{L^{\prime}}, \ldots, e_{N}^{L^{\prime}}, e_{1}^{T}, \ldots, e_{n}^{T}\right\}$ of $W$ such that the first $l$ vectors form a basis of $L$, the following $N$ vectors form a basis of a subspace $L^{\prime}$ complementary to $L$ in $V$ and the last $n$ vectors form a basis of a subspace $H$ complementary to $V$ in $W$, which is isomorphic to $T$. Then in terms of the dual basis $\left\{e_{L}^{1}, \ldots, e_{L}^{l}, e_{L^{\prime}}^{1}, \ldots, e_{L^{\prime}}^{N}, e_{T}^{1}, \ldots, e_{T}^{n}\right\}$ of $W^{*}$, we conclude that $\left\{e_{L^{\prime}}^{i_{1}} \wedge \ldots \wedge e_{L^{\prime}}^{i_{s}} \wedge e_{T}^{\mu_{1}} \wedge \ldots \wedge e_{T}^{\mu_{k-s}} \mid 0 \leqslant s \leqslant r-1,1 \leqslant i_{1}<\ldots<i_{s} \leqslant N\right.$, $\left.1 \leqslant \mu_{1}<\ldots<\mu_{k-s} \leqslant n\right\}$ is a basis of $\bigwedge_{r-1}^{k} L^{\perp}$.

The relation between multilagrangian and polylagrangian forms is established through the symbol:

Theorem 4 Let $W, V$ and $T$ be finite-dimensional vector spaces related by the short exact sequence (41), with $n=\operatorname{dim} T$, let $\omega$ be a non-vanishing $(k+1-r)$-horizontal $(k+1)$-form on $W$, where $1 \leqslant r \leqslant k+1$ and $k+1-r \leqslant n$, and let $\hat{\omega}$ be its symbol, 
which is a $\bigwedge^{k+1-r} T^{*}$-valued $r$-form on $V$. Suppose that $\omega$ is multilagrangian, with multilagrangian subspace $L$. Then $\hat{\omega}$ is polylagrangian, with polylagrangian subspace $L$, and

$$
\operatorname{ker} \omega \subset \operatorname{ker} \hat{\omega} \text {. }
$$

If $\omega$ is multipresymplectic, then $\hat{\omega}$ is polypresymplectic, with

$$
\operatorname{dim} \operatorname{ker} \hat{\omega}-\operatorname{dim} \operatorname{ker} \omega \leqslant 1 \text {. }
$$

Proof: $\quad$ Fixing an arbitrary horizontal subspace $H$ of $W$ and using the direct decompositions $W=V \oplus H$ and $W^{*}=H^{\perp} \oplus V^{\perp}$, with $H^{\perp} \cong V^{*}$ and $V^{\perp} \cong H^{*}$, we note that in order to show that $L$ is polylagrangian with respect to $\hat{\omega}$, we must establish the equality

$$
\hat{\omega}^{b}(L) \cong \bigwedge^{r-1}\left(L^{\perp} \cap H^{\perp}\right) \otimes \bigwedge^{k+1-r} T^{*} .
$$

To do so, we use the canonical isomorphism $\bigwedge^{k+1-r} T^{*} \cong \bigwedge^{k+1-r} W^{*}=\bigwedge^{k+1-r} V^{\perp}$ and the inclusion $V^{\perp} \subset L^{\perp}$, together with the fact that the space $\bigwedge^{r-1}\left(L^{\perp} \cap H^{\perp}\right) \otimes \bigwedge^{k+1-r} V^{\perp}$ is generated by elements which can be written in the form

$$
\hat{\alpha}=\left(w_{1}^{*} \wedge \ldots \wedge w_{r-1}^{*}\right) \otimes\left(w_{r}^{*} \wedge \ldots \wedge w_{k}^{*}\right) \quad \text { where } \quad \alpha=w_{1}^{*} \wedge \ldots \wedge w_{k}^{*}
$$

with $w_{1}^{*}, \ldots, w_{r-1}^{*} \in L^{\perp} \cap H^{\perp}$ and $w_{r}^{*}, \ldots, w_{k}^{*} \in V^{\perp} \subset L^{\perp}$. Since $L$ is multilagrangian with respect to $\omega$, there is a vector $u \in L$ such that $\alpha=\mathrm{i}_{u} \omega$ and so $\hat{\alpha}=\mathrm{i}_{u} \hat{\omega}$, showing that $L$ is polylagrangian with respect to $\hat{\omega}$. The inclusion (58) is obvious from the definition (46) or (47), and the estimate (59) follows by observing that the linear map $\omega^{b}: V \rightarrow \bigwedge_{r-1}{ }^{k} W^{*}$ induces an injective linear map from $V / \operatorname{ker} \omega$ to $\bigwedge_{r-1}^{k} W^{*}$ that takes ker $\hat{\omega} / \operatorname{ker} \omega$ into $\bigwedge_{r-2}^{k} W^{*}$, and this space is one-dimensional when $\omega$ is multipresymplectic.

In particular, we note that when $\omega$ is multisymplectic, there are two possibilities: either $\hat{\omega}$ is already non-degenerate or else $\hat{\omega}$ has a one-dimensional kernel. Both cases do arise in practice, so all that can be said in general about the relation between multisymplectic and polysymplectic forms (in addition to the last statement of Theorem 4 is that the symbol $\hat{\omega}$ of a multisymplectic form $\omega$ on $W$ induces a polysymplectic form on the quotient space $\hat{W}=W /$ ker $\hat{\omega}$, which by abuse of notation will again be denoted by $\hat{\omega}$.

Before going on to explore general consequences of the relation between multilagrangian and polylagrangian forms that we have just established, let us pause for a moment to comment on the situations encountered when the parameter $r$ is assumed to take on one of its extreme values. On the one hand, when $r=k+1$, the horizontality condition becomes void, the choice of $V$ and $T$ becomes irrelevant, the space $\bigwedge^{k+1-r} T^{*}$ is one-dimensional and the form $\omega$ coincides with its symbol $\hat{\omega}$. This is, once again, the particular case that has been studied before in the literature [8-10] under the label "multisymplectic forms" (a terminology that we propose to abandon since we use this term in a different sense; see Definition 3 above). Thus it becomes clear that these "multisymplectic 
forms" appear as the intersection between polylagrangian and multilagrangian forms: they are polylagrangian without being vector-valued as well as multilagrangian without being horizontal. On the other hand, note that we have already excluded the case $r=0$, where the $(k+1)$-form $\omega$ is fully horizontal (i.e., $(k+1)$-horizontal), since this situation can be reduced to the previous one if we substitute $W$ by $T$, but even the next case $r=1$, where the $(k+1)$-form $\omega$ is $k$-horizontal, is essentially trivial, since every $k$-horizontal multilagrangian $(k+1)$-form has rank 0 and multilagrangian subspace $V$. (In fact, the condition that $\omega$ should be $k$-horizontal is equivalent to the condition that $V$ should be isotropic, and in this case, $V$ does satisfy the remaining criteria of Proposition 5.)

Another fundamental property of multilagrangian forms that can be derived from the corresponding property for polylagrangian forms is that the multilagrangian subspace has a particular type of direct complement.

Theorem 5 Let $W, V$ and $T$ be finite-dimensional vector spaces related by the short exact sequence (41), with $n=\operatorname{dim} T$, and let $\omega$ be a multilagrangian $(k+1)$-form on $W$ of rank $N$ and horizontality degree $k+1-r$, where $1 \leqslant r \leqslant k+1$ and $k+1-r \leqslant n$, and with multilagrangian subspace $L$. Then there exists a $k$-isotropic subspace $F$ of $W$ such that the intersection $E=V \cap F$ is an $(r-1)$-isotropic subspace of $V$ and

$$
W=F \oplus L \quad, \quad V=E \oplus L .
$$

Proof: $\quad$ First we construct an $(r-1)$-isotropic subspace $E$ of $V$ of dimension $N$ which is complementary to $L$ in $V$. If $r=1$ there is nothing to prove since in this case the vertical subspace $V$ is isotropic and so we have $L=V, E=\{0\}$ and $N=0$. If $r>1$ we apply Theorem 2 to the symbol $\hat{\omega}$ of $\omega$ to conclude that there is a subspace $E$ of $V$ of dimension $N$ which is complementary to $L$ in $V$ and is $(r-1)$-isotropic with respect to $\hat{\omega}$. Now taking into account that the whole vertical subspace $V$ is $r$-isotropic with respect to $\omega$, it follows that $E$ is $(r-1)$-isotropic with respect to $\omega$ as well. - Now let $F_{0}$ be a subspace of $W$ of dimension $N+n^{\prime}$ which is $k$-isotropic with respect to $\omega$ and such that $F_{0} \cap V=E$. (For instance, if $n^{\prime}=0, F_{0}=E$ ). If $n^{\prime}=n$, we are done. Otherwise, choose a basis $\left\{e_{1}^{E}, \ldots, e_{N}^{E}, e_{1}, \ldots, e_{n}\right\}$ of a subspace of $W$ complementary to $L$ such that the first $N$ vectors constitute a basis of $E$ and the first $N+n^{\prime}$ vectors constitute a basis of $F_{0}$, and denote the corresponding dual basis of $L^{\perp}$ by $\left\{e_{E}^{1}, \ldots, e_{E}^{N}, e^{1}, \ldots, e^{n}\right\}$. We shall prove that there exists a vector $u \in W \backslash\left(F_{0} \oplus L\right)$ such that the subspace $F_{1}$ spanned by $u$ and $F_{0}$ is $k$-isotropic and satisfies $F_{1} \cap V=E$; then since $\operatorname{dim} F_{1}=N+n^{\prime}+1$, the statement of the theorem follows by induction. To this end, choose any subspace $L^{\prime}$ of $L$ complementary to ker $\omega$ and use the fact that $L$ is multilagrangian to conclude that there exists a unique basis

$$
\left\{e^{i_{1} \ldots i_{s} ; \mu_{1} \ldots \mu_{k-s}} \mid 0 \leqslant s \leqslant r-1, \begin{array}{c}
1 \leqslant i_{1}<\ldots<i_{s} \leqslant N \\
1 \leqslant \mu_{1}<\ldots<\mu_{k-s} \leqslant n
\end{array}\right\}
$$

of $L^{\prime}$ such that

$$
\omega_{V}^{b}\left(e^{i_{1} \ldots i_{s} ; \mu_{1} \ldots \mu_{k-s}}\right)=e_{E}^{i_{1}} \wedge \ldots \wedge e_{E}^{i_{s}} \wedge e^{\mu_{1}} \wedge \ldots \wedge e^{\mu_{k+1-s}} .
$$


Thus, for $0 \leqslant s, t \leqslant r-1,1 \leqslant i_{1}<\ldots<i_{s} \leqslant N, 1 \leqslant j_{1}<\ldots<j_{t} \leqslant N$, $1 \leqslant \mu_{1}<\ldots<\mu_{k-s} \leqslant n, 1 \leqslant \nu_{1}<\ldots<\nu_{k-t} \leqslant n$, we have

$$
\omega\left(e^{i_{1} \ldots i_{s} ; \mu_{1} \ldots \mu_{k-s}}, e_{j_{1}}^{E}, \ldots, e_{j_{t}}^{E}, e_{\nu_{1}}, \ldots, e_{\nu_{k-t}}\right)=\left\{\begin{array}{ccc}
0 & \text { if } & s \neq t \\
\delta_{j_{1}}^{i_{1}} \ldots \delta_{j_{s}}^{i_{s}} \delta_{\nu_{1}}^{\mu_{1}} \ldots \delta_{\nu_{k-s}}^{\mu_{k-s}} & \text { if } & s=t
\end{array}\right\} .
$$

Therefore, the vector

$$
u=e_{n^{\prime}+1}-\sum_{s=0}^{r-1} \frac{1}{s !} \frac{1}{(k-s) !} \omega\left(e_{n^{\prime}+1}, e_{i_{1}}^{E}, \ldots, e_{i_{s}}^{E}, e_{\mu_{1}}, \ldots, e_{\mu_{k-s}}\right) e^{i_{1} \ldots i_{s} ; \mu_{1} \ldots \mu_{k-s}}
$$

does not belong to the subspace $F_{0} \oplus L$ and, for $0 \leqslant t \leqslant r-1,1 \leqslant j_{1}<\ldots<j_{t} \leqslant N$ and $1 \leqslant \nu_{1}<\ldots<\nu_{k-t} \leqslant n$, satisfies

$$
\begin{aligned}
& \omega\left(u, e_{j_{1}}^{E}, \ldots, e_{j_{t}}^{E}, e_{\nu_{1}}, \ldots, e_{\nu_{k-t}}\right) \\
& =\omega\left(e_{n^{\prime}+1}, e_{j_{1}}^{E}, \ldots, e_{j_{t}}^{E}, e_{\nu_{1}}, \ldots, e_{\nu_{k-t}}\right) \\
& \quad-\sum_{s=0}^{r-1} \frac{1}{s ! \frac{1}{(k-s) !}} \omega\left(e_{n^{\prime}+1}, e_{i_{1}}^{E}, \ldots, e_{i_{s}}^{E}, e_{\mu_{1}}, \ldots, e_{\mu_{k-s}}\right) \\
& \quad \times \omega\left(e^{i_{1} \ldots i_{s} ; \mu_{1} \ldots \mu_{k-s}}, e_{j_{1}}^{E}, \ldots, e_{j_{t}}^{E}, e_{\nu_{1}}, \ldots, e_{\nu_{k-t}}\right) \\
& =0 .
\end{aligned}
$$

which implies that since the subspace $F_{0}$ spanned by $e_{1}^{E}, \ldots, e_{N}^{E}, e_{1}, \ldots, e_{n^{\prime}}$ is $k$-isotropic, the subspace $F_{1}$ spanned by $e_{1}^{E}, \ldots, e_{N}^{E}, e_{1}, \ldots, e_{n^{\prime}}$ and $u$ is so as well.

Example 2 (The canonical form) Let $F$ be a vector space of dimension $N+n$ and $E$ be a fixed $N$-dimensional subspace of $F$. Denoting the $n$-dimensional quotient space $F / E$ by $T$ and the canonical projection of $F$ onto $T$ by $\rho$, we obtain the following exact sequence of vector spaces:

$$
0 \longrightarrow E \longrightarrow F \stackrel{\rho}{\longrightarrow} T \longrightarrow 0
$$

Set

$$
W_{0}=F \oplus \bigwedge_{r-1}^{k} F^{*} \quad, \quad V_{0}=E \oplus \bigwedge_{r-1}^{k} F^{*}, \quad \pi_{0}=\rho \circ \operatorname{pr}_{1},
$$

where $\operatorname{pr}_{1}: W_{0} \rightarrow F$ is the canonical projection, which leads us to the following exact sequence of vector spaces:

$$
0 \longrightarrow V_{0} \longrightarrow W_{0} \stackrel{\pi_{0}}{\longrightarrow} T \longrightarrow 0 \text {. }
$$

The canonical multilagrangian form of rank $N$ and horizontality degree $k+1-r$ is the $(k+1-r)$-horizontal $(k+1)$-form $\omega_{0}$ on $W_{0}$ defined by

$$
\omega_{0}\left(\left(u_{0}, \omega_{0}\right), \ldots,\left(u_{k}, \omega_{k}\right)\right)=\sum_{i=0}^{k}(-1)^{i} \omega_{i}\left(u_{0}, \ldots, \widehat{u}_{i}, \ldots, u_{k}\right) .
$$

If $k=n$ and $r=2$ we shall call $\omega_{0}$ the canonical multisymplectic form. 
It is a straightforward exercise to show that $\omega_{0}$ is $(k+1-r)$-horizontal and is degenerate when $r=1$, with

$$
\text { ker } \omega_{0}=E \quad \text { if } r=1 \text {, }
$$

but is non-degenerate when $r>1$. In what follows, we shall assume that $N>0$, since when $E=\{0\}$, we are back to the polylagrangian case on $T \oplus \bigwedge^{k} T^{*}$, with $\hat{T}=\mathbb{R}$, which has already been studied in Refs [8-10]. For the same reason, we shall also assume that $r>1$, since for $r=1$ we have $\bigwedge_{0}^{k} F^{*} \cong \bigwedge^{k} T^{*}$, so that after passing to the quotient by the kernel of $\omega_{0}$, we are once again back to the polylagrangian case on $T \oplus \Lambda^{k} T^{*}$, with $\hat{T}=\mathbb{R}$. Then considering $F$ and

$$
L=\bigwedge_{r-1}^{k} F^{*}
$$

as subspaces of $W_{0}$, we have the direct decompositions $W_{0}=F \oplus L$ and $V_{0}=E \oplus L$ where

$$
L \text { is multilagrangian , } F \text { is } k \text {-isotropic , } E \text { is }(r-1) \text {-isotropic . }
$$

In terms of bases, let $\left\{e_{i}, e_{\mu} \mid 1 \leqslant i \leqslant N, 1 \leqslant \mu \leqslant n\right\}$ be a basis of $F$ with dual basis $\left\{e^{i}, e^{\mu} \mid 1 \leqslant i \leqslant N, 1 \leqslant \mu \leqslant n\right\}$ of $F^{*}$ such that $\left\{e_{i} \mid 1 \leqslant i \leqslant N\right\}$ is a basis of $E$ and $\left\{e_{\mu} \mid 1 \leqslant \mu \leqslant n\right\}$ is a basis of a subspace $H$ of $F$ complementary to $E$, isomorphic to $T$. For $0 \leqslant s \leqslant r, 1 \leqslant i_{1}<\ldots<i_{s} \leqslant N$ and $1 \leqslant \mu_{1}<\ldots<\mu_{k-s} \leqslant N$, define

$$
\begin{aligned}
& e_{i_{1} \ldots i_{s} ; \mu_{1} \ldots \mu_{k-s}}=e_{i_{1}} \wedge \ldots \wedge e_{i_{s}} \wedge e_{\mu_{1}} \wedge \ldots \wedge e_{\mu_{k-s}} \\
& e^{i_{1} \ldots i_{s} ; \mu_{1} \ldots \mu_{k-s}}=e^{i_{1}} \wedge \ldots \wedge e^{i_{s}} \wedge e^{\mu_{1}} \wedge \ldots \wedge e^{\mu_{k-s}}
\end{aligned}
$$

This provides a basis

$$
\left\{e_{i}, e_{\mu}, e^{i_{1} \ldots i_{s} ; \mu_{1} \ldots \mu_{k-s}} \mid 0 \leqslant s \leqslant r-1, \begin{array}{c}
1 \leqslant i \leqslant N, 1 \leqslant i_{1}<\ldots<i_{s} \leqslant N \\
1 \leqslant \mu \leqslant n, 1 \leqslant \mu_{1}<\ldots<\mu_{k-s} \leqslant n
\end{array}\right\}
$$

of $W_{0}$ with dual basis

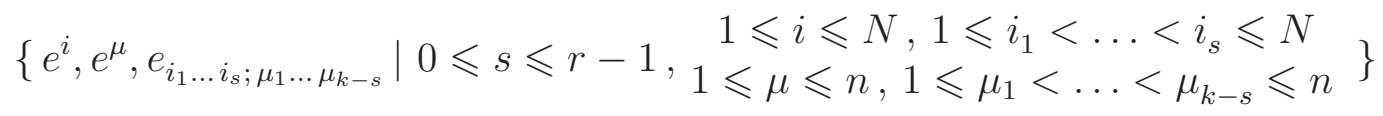

of $W_{0}^{*}$, both of which we shall refer to as a canonical basis or Darboux basis, such that

$$
\omega_{0}=\sum_{s=0}^{r-1} \frac{1}{s !} \frac{1}{(k-s) !} e_{i_{1} \ldots i_{s} ; \mu_{1} \ldots \mu_{k-s}} \wedge e^{i_{1}} \wedge \ldots \wedge e^{i_{s}} \wedge e^{\mu_{1}} \wedge \ldots \wedge e^{\mu_{k-s}} .
$$

and for the symbol

$$
\hat{\omega}_{0}=\frac{1}{(r-1) !} \frac{1}{(k+1-r) !}\left(e_{i_{1} \ldots i_{r-1} ; \mu_{1} \ldots \mu_{k+1-r}} \wedge e^{i_{1}} \wedge \ldots \wedge e^{i_{r-1}}\right) \otimes\left(e^{\mu_{1}} \wedge \ldots \wedge e^{\mu_{k+1-r}}\right) .
$$


Now it is easy to derive the algebraic Darboux theorem for general multilagrangian forms: let $\left\{e_{i}, e_{\mu} \mid 1 \leqslant i \leqslant N, 1 \leqslant \mu \leqslant n\right\}$ be a basis of a $k$-isotropic subspace $F$ complementary to $L$ in $W$, with dual basis $\left\{e^{i}, e^{\mu} \mid 1 \leqslant i \leqslant N, 1 \leqslant \mu \leqslant n\right\}$ of $L^{\perp} \cong F^{*}$, such that $\left\{e_{i} \mid 1 \leqslant i \leqslant N\right\}$ is a basis of the $(r-1)$-isotropic subspace $E=V \cap F$ which is complementary to $L$ in $V$. Choosing any subspace $L^{\prime}$ of $L$ complementary to ker $\omega$ and taking into account the identity (피), we define a basis

$$
\left\{e^{i_{1} \ldots i_{s} ; \mu_{1} \ldots \mu_{k-s}} \mid 0 \leqslant s \leqslant r-1, \begin{array}{c}
1 \leqslant i_{1}<\ldots<i_{s} \leqslant N \\
1 \leqslant \mu_{1}<\ldots<\mu_{k-s} \leqslant n
\end{array}\right\}
$$

of $L^{\prime}$ by

$$
\omega_{V}^{b}\left(e^{i_{1} \ldots i_{s} ; \mu_{1} \ldots \mu_{k-s}}\right)=e^{i_{1}} \wedge \ldots \wedge e^{i_{s}} \wedge e^{\mu_{1}} \wedge \ldots \wedge e^{\mu_{k+1-s}} .
$$

It is easy to see that the union of this basis with that of $F$ gives a canonical basis of $W$ (or more precisely, of $F \oplus L^{\prime}$, which is a subspace of $W$ complementary to ker $\omega$ ). Thus we have proved 10

\section{Theorem 6 (Darboux theorem for multilagrangian vector spaces)}

Every multilagrangian vector space admits a canonical basis.

\section{Cartan calculus and the symbol}

In order to extend the structures studied in the previous two sections and, in particular, the concept of symbol that interrelates them, from a purely algebraic setting to the realm of differential geometry, we shall need a variant of Cartan's calculus, which in its standard formulation deals with differential forms on manifolds, to handle vertical differential forms on total spaces of fiber bundles.

Let $P$ be a fiber bundle over a base manifold $M$, with projection $\pi: P \rightarrow M$. Then the vector bundle $\bigwedge_{s}^{r} T^{*} P$ over $P$ whose fiber at any point $p$ in $P$ is the space $\bigwedge_{s}^{r} T_{p}^{*} P$ of $(r-s)$-horizontal $r$-forms on the tangent space $T_{p} P$ to $P$ at $p$ is called the bundle of $(r-s)$-horizontal $r$-forms on $P$, and its sections are called $(r-s)$-horizontal differential $r$-forms or simply $(r-s)$-horizontal $r$-forms on $P$; the space of such forms will be denoted by $\Omega_{s}^{r}(P)$. Similarly, assuming in addition that $\hat{T}$ is a vector bundle over the same base manifold $M$, with projection $\hat{\tau}: \hat{T} \rightarrow M$, and denoting the pull-back of $\hat{T}$ to $P$ by $\pi^{*} \hat{T}$ and the vertical bundle of $P$ by $V P$ (both are vector bundles over $P$ ), the vector bundle $\bigwedge^{r} V^{*} P \otimes \pi^{*} \hat{T}$ over $P$ is called the bundle of vertical $r$ forms on $P$, and its sections are called vertical differential $r$-forms or simply vertical $r$-forms on $P$, with values or coefficients in $\pi^{*} \hat{T}$ or, by abuse of language, in $\hat{T}$ : the space of such forms will be denoted by $\Omega_{V}^{r}\left(P ; \pi^{*} \hat{T}\right)$. Finally, the sections of the vertical

\footnotetext{
${ }^{10}$ Once again, the inductive construction of a $k$-isotropic subspace $F$ complementary to the multilagrangian subspace $L$, as presented in the proof of Theorem 5. provides an explicit iteration procedure for building multilagrangian bases in a way similar to the well known Gram-Schmidt orthogonalization process.
} 
bundle $V P$ itself are called vertical vector fields or simply vertical fields on $P$ : the space of such fields will be denoted by $\mathfrak{X}_{V}(P)$. Obviously, $\Omega_{s}^{r}(P), \Omega_{V}^{r}\left(P ; \pi^{*} \hat{T}\right)$ and $\mathfrak{X}_{V}(P)$ are (locally finite) modules over the algebra $\mathfrak{F}(P)$ of functions on $P$.

It should be noted that speaking of vertical forms constitutes another abuse of language because these "forms" are really equivalence classes of differential $r$-forms on $P$ : $\Omega_{V}^{r}\left(P ; \pi^{*} \hat{T}\right)$ is not a subspace of the space $\Omega^{r}\left(P ; \pi^{*} \hat{T}\right)$ of all differential $r$-forms on $P$ but rather its quotient space

$$
\Omega_{V}^{r}\left(P ; \pi^{*} \hat{T}\right)=\Omega^{r}\left(P ; \pi^{*} \hat{T}\right) / \Omega_{r-1}^{r}\left(P ; \pi^{*} \hat{T}\right)
$$

by the subspace $\Omega_{r-1}^{r}\left(P ; \pi^{*} \hat{T}\right)$ of all 1-horizontal differential $r$-forms on $P$.

An interesting aspect of this construction is that it is possible to develop a variant of the usual Cartan calculus for differential forms on the manifold $P$ in which vector fields on $P$ are replaced by vertical fields $X$ on $P$ and differential forms on $P$ (taking values in some fixed vector space) are replaced by vertical differential forms $\alpha$ on $P$ (taking values in some fixed vector bundle over the base manifold), in such a way that all operations of this calculus such as exterior multiplication, contraction, Lie derivative and exterior derivative continue to be well defined and to satisfy the standard rules. (See [17, Vol. 1, Problem 8, p. 313] for the special case where the vector bundle of coefficients is the trivial line bundle $M \times \mathbb{R}$.) Here, we shall only need the vertical exterior derivative

$$
\begin{array}{ccc}
d_{V}: \Omega_{V}^{r}\left(P ; \pi^{*} \hat{T}\right) & \longrightarrow & \Omega_{V}^{r+1}\left(P ; \pi^{*} \hat{T}\right) \\
\alpha & \longmapsto & d_{V} \alpha
\end{array}
$$

which is defined by exactly the same formula as in the standard case, namely

$$
\begin{aligned}
d_{V} \alpha\left(X_{0}, \ldots, X_{r}\right)= & \sum_{i=0}^{r}(-1)^{i} X_{i} \cdot\left(\alpha\left(X_{0}, \ldots, \hat{X}_{i}, \ldots, X_{r}\right)\right) \\
& +\sum_{0 \leqslant i<j \leqslant r}(-1)^{i+j} \alpha\left(\left[X_{i}, X_{j}\right], X_{0}, \ldots, \hat{X}_{i}, \ldots, \hat{X}_{j}, \ldots, X_{r}\right)
\end{aligned}
$$

where $X_{0}, X_{1}, \ldots, X_{r} \in \mathfrak{X}_{V}(P)$ : this makes sense since $V P$ is an involutive distribution on $P$, provided we correctly define the vertical directional derivative

$$
\begin{array}{cl}
\mathfrak{X}_{V}(P) \times \Gamma\left(\pi^{*} \hat{T}\right) & \longrightarrow \Gamma\left(\pi^{*} \hat{T}\right) \\
(X, \varphi) & \longmapsto
\end{array}
$$

as an $\mathbb{R}$-bilinear operator which is $\mathfrak{F}(P)$-linear in the first entry and satisfies a Leibniz rule in the second entry,

$$
X \cdot(f \varphi)=(X \cdot f) \varphi+f(X \cdot \varphi) .
$$

Explicitly, for $X \in \mathfrak{X}_{V}(P)$ and $\varphi \in \Gamma\left(\pi^{*} \hat{T}\right), X \cdot \varphi \in \Gamma\left(\pi^{*} \hat{T}\right)$ is defined as the standard directional derivative of vector valued functions along the fibers, that is, for 
any point $m$ in $M,\left.(X \cdot \varphi)\right|_{P_{m}} \in C^{\infty}\left(P_{m}, \hat{T}_{m}\right)$ is given in terms of $\left.X\right|_{P_{m}} \in \mathfrak{X}\left(P_{m}\right)$ and $\left.\varphi\right|_{P_{m}} \in C^{\infty}\left(P_{m}, \hat{T}_{m}\right)$ by

$$
\left.(X \cdot \varphi)\right|_{P_{m}}=\left.\left.X\right|_{P_{m}} \cdot \varphi\right|_{P_{m}} .
$$

Since the Lie bracket is natural under restriction to submanifolds, we have

$$
X \cdot(Y \cdot \varphi)-Y \cdot(X \cdot \varphi)=[X, Y] \cdot \varphi \quad \text { for } X, Y \in \mathfrak{X}_{V}(P), \varphi \in \Gamma\left(\pi^{*} \hat{T}\right),
$$

which implies that $d_{V}^{2}=0$. On the other hand, sections $\varphi$ of $\pi^{*} \hat{T}$ obtained from sections $\hat{t}$ of $\hat{T}$ by composing with $\pi$ are constant along the fibers and hence their vertical directional derivative vanishes:

$$
X \cdot(\hat{t} \circ \pi)=0 \quad \text { for } X \in \mathfrak{X}_{V}(P), \hat{t} \in \Gamma(\hat{T}) .
$$

In the same way, substituting $\hat{T}$ by $\hat{T}^{*}$, we get

$$
X \cdot\left(\hat{t}^{*} \circ \pi\right)=0 \quad \text { for } X \in \mathfrak{X}_{V}(P), \hat{t}^{*} \in \Gamma\left(\hat{T}^{*}\right),
$$

which means that

$$
X \cdot\left\langle\hat{t}^{*} \circ \pi, \varphi\right\rangle=\left\langle\hat{t}^{*} \circ \pi,(X \cdot \varphi)\right\rangle \quad \text { for } X \in \mathfrak{X}_{V}(P), \varphi \in \Gamma\left(\pi^{*} \hat{T}\right) .
$$

More generally, given a $\pi^{*} \hat{T}$-valued vertical $r$-form $\hat{\alpha}$ on $P$ and a section $\hat{t}^{*}$ of the dual vector bundle $\hat{T}^{*}$ of $\hat{T}$, we define the projection of $\hat{\alpha}$ along $\hat{t}^{*}$ to be the ordinary vertical $r$-form $\hat{\alpha}_{\hat{t}^{*}}$ on $P$ given by 11

$$
\hat{\alpha}_{\hat{t}^{*}}(p)=\left\langle\hat{t}^{*}(\pi(p)), \hat{\alpha}(p)\right\rangle \quad \text { for } p \in P,
$$

and obtain

$$
d_{V} \hat{\alpha}_{\hat{t}^{*}}=\left(d_{V} \hat{\alpha}\right)_{\hat{t}^{*}} .
$$

Hence, if $\hat{\alpha}$ is closed, $\hat{\alpha}_{\hat{t}^{*}}$ will be closed as well, and a standard argument shows that, in this case, the kernel of $\hat{\alpha}$ and of $\hat{\alpha}_{\hat{t}^{*}}$, given by

$$
\operatorname{ker}_{p} \hat{\alpha}=\operatorname{ker} \hat{\alpha}_{p}=\left\{u_{p} \in V_{p} P \mid \mathrm{i}_{u_{p}} \hat{\alpha}_{p}=0\right\} \quad \text { for } p \in P
$$

and

$$
\operatorname{ker}_{p} \hat{\alpha}_{\hat{t}^{*}}=\operatorname{ker}\left(\hat{\alpha}_{\hat{t}^{*}}\right)_{p}=\left\{u_{p} \in V_{p} P \mid \mathrm{i}_{u_{p}}\left(\hat{\alpha}_{\hat{t}^{*}}\right)_{p}=0\right\} \quad \text { for } p \in P,
$$

respectively, define involutive distributions on $P$, provided they have constant dimension.

With these tools at our disposal, we can now formulate the construction of the symbol in the differential geometric setting. To deal directly with the case of interest, suppose that $\omega$ is a $(k+1-r)$-horizontal $(k+1)$-form on $P$, where $1 \leqslant r \leqslant k+1$ and $k+1-r \leqslant n$, that is,

$$
\omega \in \Omega_{r}^{k+1}(P)=\Gamma\left(\bigwedge_{r}^{k+1} T^{*} P\right) .
$$

\footnotetext{
${ }^{11}$ Here, it is important that $\hat{t}^{*}$ be a section of $\hat{T}^{*}$ and not of $\pi^{*} \hat{T}^{*}$.
} 
Explicitly, the horizontality condition means that contraction of $\omega$ with more than $r$ vertical fields on $P$ gives zero. Then the symbol $\hat{\omega}$ of $\omega$, whose value at every point $p$ of $P$ is defined to be the symbol $\hat{\omega}_{p}$ of $\omega_{p}$, is a vertical $r$-form on $P$ taking values in the vector bundle $\pi^{*}\left(\bigwedge^{k+1-r} T^{*} M\right)$, that is,

$$
\hat{\omega} \in \Omega_{V}^{r}\left(P, \pi^{*}\left(\bigwedge^{k+1-r} T^{*} M\right)\right)=\Gamma\left(\bigwedge^{r} V^{*} P \otimes \pi^{*}\left(\bigwedge^{k+1-r} T^{*} M\right)\right) .
$$

Using the canonical isomorphism $\pi^{*}\left(\bigwedge^{k+1-r} T^{*} M\right) \cong \bigwedge_{0}^{k+1-r} T^{*} P$ of vector bundles over $P$ as an identification, we have

$$
\hat{\omega}\left(X_{1}, \ldots, X_{r}\right)=\mathrm{i}_{X_{1}} \ldots \mathrm{i}_{X_{r}} \omega \quad \text { for } X_{1}, \ldots, X_{r} \in \mathfrak{X}_{V}(P) .
$$

More explicitly, using the horizontal lift

$$
\begin{aligned}
\mathfrak{X}(M) & \longrightarrow \mathfrak{X}(P) \\
\xi & \longmapsto \xi^{H}
\end{aligned}
$$

of vector fields induced by some fixed connection on $P$, we have

$$
\begin{gathered}
\hat{\omega}\left(X_{1}, \ldots, X_{r}\right) \cdot\left(\xi_{1} \circ \pi, \ldots, \xi_{k+1-r} \circ \pi\right)=\omega\left(X_{1}, \ldots, X_{r}, \xi_{1}^{H}, \ldots, \xi_{k+1-r}^{H}\right) \\
\text { for } X_{1}, \ldots, X_{r} \in \mathfrak{X}_{V}(P), \xi_{1}, \ldots, \xi_{k+1-r} \in \mathfrak{X}(M),
\end{gathered}
$$

where it should be noted that although composition of vector fields on $M$ with the projection $\pi$ provides only a subspace of the vector space of all sections of the pull-back $\pi^{*} T M$ of $T M$ by $\pi$, this formula is sufficient to fix the value of $\hat{\omega}$ at each point $p$ of $P$.

Theorem $7 \quad$ With the same notations as above, suppose that the form $\omega$ satisfies

$$
d \omega \in \Omega_{r}^{k+2}(P)=\Gamma\left(\bigwedge_{r}^{k+2} T^{*} P\right),
$$

i.e., $d \omega$ is $(k+2-r)$-horizontal. Then the form $\hat{\omega}$ is vertically closed:

$$
d_{V} \hat{\omega}=0 \text {. }
$$

In particular, for $\hat{\omega}$ to be vertically closed, it is sufficient (but not necessary) that $\omega$ be closed.

Proof: Let $X_{0}, \ldots, X_{r}$ be vertical fields on $P$ and $\xi_{1}, \ldots, \xi_{k+1-r}$ be vector fields on $M$, and denote the horizontal lifts of the latter with respect to some fixed connection on $P$ by $\xi_{1}^{H}, \ldots, \xi_{k+1-r}^{H}$, respectively. Then we have the relation

$$
d \omega\left(X_{0}, \ldots, X_{r}, \xi_{1}^{H}, \ldots, \xi_{k+1-r}^{H}\right)=\left(d_{V} \hat{\omega}\left(X_{0}, \ldots, X_{r}\right)\right)\left(\xi_{1} \circ \pi, \ldots, \xi_{k+1-r} \circ \pi\right)
$$

which can be derived from Cartan's formula for $d \omega$ and its analogue for $d_{V} \hat{\omega}$, equation (71), using that $\omega$ is a $(k+1-r)$-horizontal $(k+1)$-form and the Lie brackets $\left[X_{i}, \xi_{j}^{H}\right]$ are vertical fields, by applying equation (177) to expressions of the form $\varphi=\hat{\omega}\left(X_{0}, \ldots, \hat{X}_{i}, \ldots, X_{r}\right)$ and $\hat{t}^{*}=\xi_{1} \wedge \ldots \wedge \xi_{k+1-r}$. 


\section{Polylagrangian and multilagrangian fiber bundles}

Now we are ready to transfer the poly- and multilagrangian structures introduced in the first two sections from the algebraic to the differential geometric context. All we need to do is add the appropriate integrability condition, which is the expected one: the differential forms in question should be closed. For the sake of brevity, we begin directly with the notion of a polylagrangian fiber bundle, which formalizes the idea of a "family of polylagrangian manifolds smoothly parametrized by the points of a base manifold $M$ " and includes that of a polylagrangian manifold as a special case.

Definition 4 A polylagrangian fiber bundle is a fiber bundle $P$ over an $n$ dimensional manifold $M$ equipped with a vertical $(k+1)$-form $\hat{\omega}$ of constant rank on the total space $P$ taking values in a fixed $\hat{n}$-dimensional vector bundle $\hat{T}$ over the same manifold $M$, called the polylagrangian form along the fibers of $P$, or simply the polylagrangian form, and said to be of rank $N$, such that $\hat{\omega}$ is vertically closed,

$$
d_{V} \hat{\omega}=0
$$

and such that at every point $p$ of $P, \hat{\omega}_{p}$ is a polylagrangian form of rank $N$ on the vertical space $V_{p} P$. If the polylagrangian subspaces at the different points of $P$ fit together into a distribution $L$ on $P$ (which is contained in the vertical bundle $V P$ of $P$ ), we call it the polylagrangian distribution of $\hat{\omega}$.

When $k=1$ and $\hat{\omega}$ is non-degenerate, we say that $P$ is a polysymplectic fiber bundle and $\hat{\omega}$ is a polysymplectic form along the fibers of $P$, or simply a polysymplectic form. If the condition of non-degeneracy is dropped, we call $P$ a polypresymplectic fiber bundle and $\hat{\omega}$ a polypresymplectic form along the fibers of $P$, or simply a polypresymplectic form.

If $M$ reduces to a point, we speak of a polylagrangian manifold or poly(pre)symplectic manifold, respectively.

The notion of a multilagrangian fiber bundle is defined similarly.

Definition 5 A multilagrangian fiber bundle is a fiber bundle $P$ over an $n$ dimensional manifold $M$ equipped with a $(k+1-r)$-horizontal $(k+1)$-form $\omega$ of constant rank on the total space $P$, where $1 \leqslant r \leqslant k+1$ and $k+1-r \leqslant n$, called the multilagrangian form and said to be of rank $N$ and horizontality degree $k+1-r$, such that $\omega$ is closed,

$$
d \omega=0
$$

and such that at every point $p$ of $P, \omega_{p}$ is a multilagrangian form of rank $N$ on the tangent space $T_{p} P$. If the multilagrangian subspaces at the different points of $P$ fit together into a distribution $L$ on $P$ (which is contained in the vertical bundle VP of $P$ ), we call it the multilagrangian distribution of $\omega$. 
When $k=n, r=2$ and $\omega$ is non-degenerate, we say that $P$ is a multisymplectic fiber bundle and $\omega$ is a multisymplectic form. If the condition of non-degeneracy is dropped, we call $P$ a multipresymplectic fiber bundle and $\omega$ a multipresymplectic form.

If $M$ reduces to a point, we speak of a multilagrangian manifold.

Note that within the logic underlying this terminology, the concept of a multisymplectic manifold by itself is meaningless, since multisymplectic structures "live" on total spaces of fiber bundles over some non-trivial base space (representing space-time). The "multisymplectic manifolds" of Refs [8-10] correspond to what we call multilagrangian manifolds.

Combining Theorem 4 and Theorem [7, we obtain

Theorem 8 Let $P$ be a fiber bundle over an $n$-dimensional manifold $M$, with projection $\pi: P \rightarrow M$, let $\omega$ be $a(k+1-r)$-horizontal $(k+1)$-form of constant rank on $P$, where $1 \leqslant r \leqslant k+1$ and $k+1-r \leqslant n$, and let $\hat{\omega}$ be its symbol, which is a vertical $r$ form on $P$ taking values in the bundle of $(k+1-r)$-forms on $M$. Suppose that $\omega$ is multilagrangian, with multilagrangian distribution L. Then $\hat{\omega}$ is polylagrangian, with polylagrangian distribution $L$, and

$$
\operatorname{ker} \omega \subset \operatorname{ker} \hat{\omega} \text {. }
$$

If $\omega$ is multipresymplectic, then $\hat{\omega}$ is polypresymplectic, with

$$
\operatorname{dim} \operatorname{ker} \hat{\omega}-\operatorname{dim} \operatorname{ker} \omega \leqslant 1 \text {. }
$$

In particular, we note again that when $\omega$ is multisymplectic, there are two possibilities: either $\hat{\omega}$ is already non-degenerate or else $\hat{\omega}$ has a one-dimensional kernel. Both cases do arise in practice, so all that can be said in general about the relation between multisymplectic and polysymplectic structures on fiber bundles (in addition to the last statement of Theorem (8) is that the symbol $\hat{\omega}$ of a multisymplectic form $\omega$ on $P$ induces a polysymplectic form on the quotient bundle $\hat{P}=P / \operatorname{ker} \hat{\omega}$ (provided that this quotient is really a fiber bundle over $M$ ), which by abuse of notation will again be denoted by $\hat{\omega}$.

Remark 2 Note that when $\hat{n} \geqslant 2$, in the case of polylagrangian structures, or when $1 \leqslant r \leqslant k$, in the case of multilagrangian structures, the explicit construction of $L$ in terms of the kernels of the projections of $\hat{\omega}$ (see Theorem 11) implies that the polylagrangian or multilagrangian subspaces at the different points of $P$ do fit together to form a uniquely determined, smooth distribution $L$ on $P$. (The proof uses the fact that sums and intersections of smooth vector subbundles of a vector bundle are again smooth vector subbundles if they have constant rank and, more generally, that kernels and images of smooth vector bundle homomorphisms of constant rank are smooth vector subbundles; 
see, e.g., [19, Exercise 1.6F(c), p. 51].) But in the case of scalar polylagrangian structures $(\hat{n}=1)$ and of multilagrangian structures for which the horizontality condition is void $(r=k+1)$, such a distribution may fail to exist, even if we assume the base manifold to be trivial (i.e., $M$ reduces to a point, $n=0$ ) and the form $\hat{\omega}$ or $\omega$ to be non-degenerate. Indeed, for symplectic forms $(k=1)$ or volume forms $(N=k)$, it is easy to find examples of manifolds whose tangent bundle does not admit any (smooth) lagrangian subbundle: the simplest of them all is just the 2-sphere $S^{2}$, according to the "no hair theorem". And even in the remaining cases $(N>k>1)$, where according to Proposition 3 , the polylagrangian or multilagrangian subspaces at the different points of $P$ are unique, an explicit construction for them does not seem to be available, so we do not know whether it is true that smoothness of $\hat{\omega}$ or $\omega$ by itself implies smoothness of $L$. Thus when we simply refer to "the polylagrangian distribution $L$ " or "the multilagrangian distribution $L$ " without further specification, as will often be done in what follows, existence and smoothness of $L$ is in these cases tacitly assumed.

\section{$5 \quad$ Integrability}

A further remarkable property of polylagrangian/multilagrangian structures is that, generically, the polylagrangian/multilagrangian distribution is automatically integrable and thus gives rise to a polylagrangian/multilagrangian foliation.

Theorem 9 (Integrability Theorem) Let $P$ be a polylagrangian fiber bundle over an $n$-dimensional manifold $M$ with polylagrangian $(k+1)$-form $\hat{\omega}$ of rank $N$ taking values in a fixed $\hat{n}$-dimensional vector bundle $\hat{T}$ over the same manifold $M$. Then if

$$
\hat{n} \geqslant 3
$$

the polylagrangian distribution $L$ is integrable. Similarly, let $P$ be a multilagrangian fiber bundle over an $n$-dimensional manifold $M$ with multilagrangian $(k+1)$-form $\omega$ of rank $N$ and horizontality degree $k+1-r$, where $1 \leqslant r \leqslant k+1$ and $k+1-r \leqslant n$. Then if

$$
\left(\begin{array}{c}
n \\
k+1-r
\end{array}\right) \geqslant 3
$$

the multilagrangian distribution $L$ is integrable.

Proof: Using Theorem 8 , the second statement is easily reduced to the first. To prove this, suppose that $X$ and $Y$ are vector fields on $P$ which are sections of $L \subset V P$. Using the decomposition

$$
L=K_{0} \oplus K_{1} \oplus \ldots \oplus K_{\hat{n}},
$$

with $K_{0}=$ ker $\hat{\omega}$, as in equation (31), we can decompose $X$ and $Y$ according to

$$
X=\sum_{a=0}^{\hat{n}} X_{a} \quad, \quad Y=\sum_{b=0}^{\hat{n}} Y_{b}
$$


where $X_{a}$ and $Y_{b}$ are sections of $K_{a}$ and $K_{b}$, respectively. Using that $\hat{n} \geqslant 3$, we can for each value of $a$ and $b$ find a value $c \neq 0$ such that $c \neq a$ and $c \neq b$; then $K_{a} \subset \operatorname{ker} \omega^{c}$ and $K_{b} \subset \operatorname{ker} \omega^{c}$. Since $\omega^{c}$ is vertically closed and has constant rank, ker $\omega^{c} \subset V P$ is involutive. Therefore the vector field $\left[X_{a}, Y_{b}\right]$ is a section of ker $\omega^{c} \subset L$.

It must be emphasized that when the above inequalities are not satisfied, the statement of Theorem 9 is false, i.e., $L$ may fail to be involutive. For the multilagrangian case, it is instructive to spell out explicitly under what circumstances this may happen:

1. $r=k+1, n=0,1,2,3, \ldots$ arbitrary: this is the extreme case discussed before in which the horizontality condition is void.

2. $r=k+1-n, n=1,2,3, \ldots$ arbitrary: this includes the symplectic case, obtained by choosing $k=n+1$ and $r=2$ (i.e., $\left.\omega \in \Omega_{2}^{n+2}(P)\right)$ and supposing in addition that ker $\hat{\omega}=\{0\}$ and that $M$ is orientable, so that $\hat{\omega} \in \Omega_{V}^{2}\left(P, \pi^{*}\left(\bigwedge^{n} T^{*} M\right)\right)$ represents a "family of symplectic forms smoothly parametrized by the points of a base manifold $M$ of dimension $n$ ". Here, it is not difficult to construct examples of lagrangian distributions which are not involutive.

3. $r=k, n=2$ (the only possibility to have $\left(\begin{array}{c}n \\ k+1-r\end{array}\right) \leqslant 2$ with $1 \leqslant r \leqslant k$ and $k+1-r<n)$ : this includes the multisymplectic case over a two-dimensional base manifold $M$, obtained by choosing $r=k=n=2$. An explicit example of this situation is given in Example 5 below.

A simple example of a polysymplectic manifold with a two-dimensional non-involutive polylagrangian distribution of rank 1 is the following.

Example 3 Let $P$ be $S U(2)$ and let $\alpha \in \Omega^{1}(P, \mathfrak{s u}(2))$ be the left invariant MaurerCartan form on $P$, where $\mathfrak{s u}(2)$ is the Lie algebra of $S U(2)$. Consider the left invariant $\mathfrak{s u}(2)^{*}$-valued 2 -form $\beta$ on $P$ obtained by taking the exterior product of component forms whose values are multiplied using the commutator in $\mathfrak{s u}(2)$ and finally passing to the dual using the invariant scalar product $(.,$.$) on \mathfrak{s u}(2)$, i.e.,

$$
\langle\beta, X\rangle=\frac{1}{2}([\alpha \wedge \alpha], X) \quad \text { for } X \in \mathfrak{s u}(2) .
$$

Using the isomorphism $\mathfrak{s u}(2) \cong \mathbb{R}^{3}$ induced by employing the standard orthonormal basis $\left\{\sigma_{a} / 2 i \mid a \in\{1,2,3\}\right\}$ of $\mathfrak{s u}(2) 12$ and working in components, we see that $\alpha$ is represented

\footnotetext{
${ }^{12}$ Note that under the isomorphism $\mathfrak{s u}(2) \cong \mathbb{R}^{3}$ given by mapping the basis $\left\{\sigma_{a} / 2 i \mid a \in\{1,2,3\}\right\}$ of $\mathfrak{s u}(2)$ to the standard basis of $\mathbb{R}^{3}$, where

$$
\sigma_{1}=\left(\begin{array}{ll}
0 & 1 \\
1 & 0
\end{array}\right), \sigma_{2}=\left(\begin{array}{cc}
0 & -i \\
i & 0
\end{array}\right), \quad \sigma_{3}=\left(\begin{array}{cc}
1 & 0 \\
0 & -1
\end{array}\right)
$$

are the usual Pauli matrices, the commutator in $\mathfrak{s u}(2)$ corresponds to the vector product in $\mathbb{R}^{3}$ whereas the invariant scalar product in $\mathfrak{s u}(2)$ given by $(X, Y)=-2 \operatorname{tr}(X Y)$ corresponds to the standard scalar product in $\mathbb{R}^{3}$. With respect to this orthonormal basis, the (totally covariant) structure constants for $\mathfrak{s u}(2)$ are given by the components of the $\epsilon$-tensor, which are those of the standard volume form on $\mathbb{R}^{3}$.
} 
by a triplet of left invariant 1-forms $\alpha^{1}, \alpha^{2}, \alpha^{3}$ and $\beta$ by a triplet of left invariant 2-forms $\beta_{1}, \beta_{2}, \beta_{3}$ on $P$ such that

$$
\beta_{a}=\frac{1}{2} \epsilon_{a b c} \alpha^{b} \wedge \alpha^{c} .
$$

Moreover, taking $\hat{T}=\left(\mathbb{R}^{2}\right)^{*}$, let $e$ be a given 2-frame in $\mathbb{R}^{3}$, i.e., a given injective linear map $e$ from $\mathbb{R}^{2}$ to $\mathbb{R}^{3}$. Its transpose will be a surjective linear map (projection) pr from $\left(\mathbb{R}^{3}\right)^{*}$ to $\left(\mathbb{R}^{2}\right)^{*} 13$ and we define

$$
\hat{\omega}=\operatorname{pr} \circ \beta .
$$

In components, $e$ is represented by a triplet of vectors $e^{1}, e^{2}, e^{3}$ in $\left(\mathbb{R}^{2}\right)^{*}$ (not a basis, of course), and we have

$$
\hat{\omega}=\frac{1}{2} \epsilon_{a b c} \alpha^{a} \wedge \alpha^{b} \otimes e^{c} .
$$

It follows immediately from the Maurer-Cartan structure equations that each of the 2forms $\beta_{1}, \beta_{2}, \beta_{3}$ is closed, and hence so is $\hat{\omega}$. Moreover, it is easily verified that the image of $\mathbb{R}^{2}$ under $e$, regarded as a subspace of $\mathfrak{s u}(2) \cong \mathbb{R}^{3}$, generates a two-dimensional left invariant distribution $L$ on $P$ which is isotropic under $\hat{\omega}$ (since the expression $\epsilon_{a b c} e^{a}(u) e^{b}(v) e^{c}(w)$ vanishes identically, for any three vectors $u, v, w$ in $\mathbb{R}^{2}$ ), so $\hat{\omega}$ is polysymplectic and $L$ is the corresponding polylagrangian distribution. Of course, $L$ is not involutive.

Similar but somewhat more complicated constructions lead to examples of polysymplectic and multisymplectic fiber bundles over an arbitrary two-dimensional base manifold $M$ whose polylagrangian or multilagrangian distribution, again of rank 1 , is not involutive. All of them are principal bundles over $M$, with structure group $S U(2)$ in the polysymplectic case and $U(2)$ in the multisymplectic case, carrying an invariant polysymplectic or multisymplectic form built from data that can be interpreted in terms of concepts from Yang-Mills-Higgs field theory, namely an $S U(2)$-connection form $A$ and a Higgs field $\phi$ in the adjoint representation of $S U(2)$ in the polysymplectic case and a $U(1)$-connection form $A$ and a Higgs field $\phi$ in the truncated adjoint representation of $U(2)$ in the multisymplectic case 14 The details follow.

Example 4 Let $P$ be the total space of a principal bundle over a two-dimensional manifold $M$ with structure group $S U(2)$ and bundle projection $\pi: P \rightarrow M$ and let $A \in \Omega^{1}(P, \mathfrak{s u}(2))$ be a given connection form on $P$, where $\mathfrak{s u}(2)$ is the Lie algebra of $S U(2)$. Consider the equivariant $\mathfrak{s u}(2)^{*}$-valued 2 -form $B$ on $P$ obtained by taking the exterior

\footnotetext{
${ }^{13}$ It may seem overly pedantic not to identify the spaces $\mathbb{R}^{2}$ and $\mathbb{R}^{3}$ with their respective duals $\left(\mathbb{R}^{2}\right)^{*}$ and $\left(\mathbb{R}^{3}\right)^{*}$, but we have refrained from doing so right from the start since this turns out to facilitate the understanding of the generalizations to be discussed in the next two examples. Moreover, maintaining this distinction is not completely irrelevant since $e$ is not assumed to be isometric: the space $\mathbb{R}^{2}$ is not even supposed to carry a scalar product.

${ }^{14}$ By the truncated adjoint representation of a Lie group $G$, we mean the restriction of the adjoint representation of $G$ on its Lie algebra $\mathfrak{g}$ to its derived algebra $[\mathfrak{g}, \mathfrak{g}]$, which is an $\operatorname{Ad}(G)$-invariant subspace (in particular, an ideal) of $\mathfrak{g}$. Note that the derived algebra of $\mathfrak{u}(n)$ is $\mathfrak{s u}(n)$.
} 
product of component forms whose values are multiplied using the commutator in $\mathfrak{s u}(2)$ and finally passing to the dual using the invariant scalar product $(.,$.$) on \mathfrak{s u}(2)$, i.e.,

$$
\langle B, X\rangle=\frac{1}{2}([A \wedge A], X) \quad \text { for } X \in \mathfrak{s u}(2) \text {. }
$$

Using the isomorphism $\mathfrak{s u}(2) \cong \mathbb{R}^{3}$ as before 12 and working in components, we see that $A$ is represented by a triplet of 1 -forms $A^{1}, A^{2}, A^{3}$ and $B$ by a triplet of 2 -forms $B_{1}, B_{2}, B_{3}$ on $P$ such that

$$
B_{a}=\frac{1}{2} \epsilon_{a b c} A^{b} \wedge A^{c} .
$$

Moreover, taking $\hat{T}=T^{*} M$, let $e$ be a given 1 -form on $M$ taking values in the adjoint bundle $P \times_{S U(2)} \mathbb{R}^{3}$ which is an "immersion" in the sense that, when interpreted as a vector bundle homomorphism from $T M$ to $P \times_{S U(2)} \mathbb{R}^{3}$, it is fiberwise injective. When pulled back to $P$, e corresponds to an equivariant horizontal $\mathbb{R}^{3}$-valued 1 -form $\pi^{*} e$ on $P$ which, once again, is an "immersion" in the sense that, when interpreted as an equivariant vector bundle homomorphism from $\pi^{*}(T M)$ to $P \times \mathbb{R}^{3}$, it is fiberwise injective. Its transpose will be a fiberwise surjective equivariant vector bundle homomorphism (equivariant projection) pr from $P \times\left(\mathbb{R}^{3}\right)^{*}$ to $\pi^{*}\left(T^{*} M\right)$, and we define

$$
\hat{\omega}=\left.\operatorname{pr} \circ B\right|_{V P},
$$

where $\left.\right|_{V P}$ denotes restriction to the vertical bundle $V P$ of $P$. In components, $\pi^{*} e$ is represented by a triplet of sections $\pi^{*} e^{1}, \pi^{*} e^{2}, \pi^{*} e^{3}$ of $\pi^{*}\left(T^{*} M\right)$, and we have

$$
\hat{\omega}=\left.\left.\frac{1}{2} \epsilon_{a b c} A^{a}\right|_{V P} \wedge A^{b}\right|_{V P} \otimes \pi^{*} e^{c} .
$$

It follows immediately from the structure equation

$$
\left.d_{V} A^{a}\right|_{V P}=\left.\left(d A^{a}\right)\right|_{V P}=-\left.\left.\frac{1}{2} \epsilon_{b c}^{a} A^{b}\right|_{V P} \wedge A^{c}\right|_{V P}
$$

together with the formula $\epsilon_{a b c} \epsilon^{a}{ }_{d e}=\delta_{b d} \delta_{c e}-\delta_{b e} \delta_{c d}$, that $\hat{\omega}$ is vertically closed:

$$
\begin{aligned}
d_{V} \hat{\omega}= & \left.\left.\frac{1}{2} \epsilon_{a b c} d_{V} A^{a}\right|_{V P} \wedge A^{b}\right|_{V P} \otimes \pi^{*} e^{c}-\left.\left.\frac{1}{2} \epsilon_{a b c} A^{a}\right|_{V P} \wedge d_{V} A^{b}\right|_{V P} \otimes \pi^{*} e^{c} \\
= & -\left.\left.\left.\frac{1}{2} \epsilon_{a b c} \epsilon_{d e}^{a} A^{d}\right|_{V P} \wedge A^{e}\right|_{V P} \wedge A^{b}\right|_{V P} \otimes \pi^{*} e^{c} \\
& +\left.\left.\left.\frac{1}{2} \epsilon_{a b c} \epsilon_{d e}^{b} A^{a}\right|_{V P} \wedge A^{d}\right|_{V P} \wedge A^{e}\right|_{V P} \otimes \pi^{*} e^{c} \\
= & 0 .
\end{aligned}
$$

Moreover, it is easily verified that the image of $\pi^{*}(T M)$ under $\pi^{*} e$, when transferred from $P \times \mathbb{R}^{3}$ to $V P$ by means of the canonical isomorphism that exists between the two since $P$ is a principal bundle, constitutes a two-dimensional invariant distribution $L$ on $P$ which is isotropic under $\hat{\omega}$, so $\hat{\omega}$ is polysymplectic and $L$ is the corresponding polylagrangian distribution. Of course, $L$ is not involutive. 
Example 5 Let $P$ be the total space of a principal bundle over a two-dimensional manifold $M$ with structure group $U(2)$ and bundle projection $\pi: P \rightarrow M$ and let $A \in \Omega^{1}(P, \mathfrak{u}(2))$ be a given connection form on $P$, where $\mathfrak{u}(2)$ is the Lie algebra of $U(2)$. Consider the equivariant $\mathfrak{s u}(2)^{*}$-valued 2 -form $B$ on $P$ obtained by taking the exterior product of component forms whose values are multiplied using the commutator in $\mathfrak{u}(2)$ (which maps into the derived algebra $\mathfrak{s u}(2)$ ) and finally passing to the dual using the invariant scalar product $(.,$.$) on \mathfrak{s u}(2)$, i.e.,

$$
\langle B, X\rangle=\frac{1}{2}([A \wedge A], X) \quad \text { for } X \in \mathfrak{s u}(2) .
$$

Using the isomorphism $\mathfrak{u}(2) \cong \mathbb{R}^{4}$ induced by employing the standard orthonormal basis $\left\{\sigma_{a} / 2 i \mid a \in\{0,1,2,3\}\right\}$ of $\mathfrak{u}(2) 15$ and working in components, we see that $A$ is represented by a quartet of 1 -forms $A^{0}, A^{1}, A^{2}, A^{3}$ and $B$ by a triplet of 2-forms $B_{1}, B_{2}, B_{3}$ on $P$ such that

$$
B_{a}=\frac{1}{2} \epsilon_{a b c} A^{b} \wedge A^{c} .
$$

Moreover, let $\phi$ be a given section of the truncated adjoint bundle $P \times_{U(2)} \mathbb{R}^{3}$ which is an "immersion" in the sense that its covariant derivative $D_{A} \phi$ with respect to the given connection, which is a 1 -form on $M$ taking values in $P \times_{U(2)} \mathbb{R}^{3}$, when interpreted as a vector bundle homomorphism from $T M$ to $P \times_{U(2)} \mathbb{R}^{3}$, is fiberwise injective. When pulled back to $P, \phi$ corresponds to an equivariant $\mathbb{R}^{3}$-valued function $\pi^{*} \phi$ on $P$ and $D_{A} \phi$ corresponds to an equivariant horizontal $\mathbb{R}^{3}$-valued 1 -form $\pi^{*} D_{A} \phi=D_{A} \pi^{*} \phi$ on $P$ which, once again, is an "immersion" in the sense that, when interpreted as an equivariant vector bundle homomorphism from $\pi^{*}(T M)$ to $P \times \mathbb{R}^{3}$, it is fiberwise injective. In components, $\pi^{*} \phi$ is represented by a triplet of functions $\pi^{*} \phi^{1}, \pi^{*} \phi^{2}, \pi^{*} \phi^{3}$ and $D_{A} \pi^{*} \phi$ by a triplet of horizontal 1-forms $D_{A} \pi^{*} \phi^{1}, D_{A} \pi^{*} \phi^{2}, D_{A} \pi^{*} \phi^{3}$ on $P$, where

$$
D_{A} \pi^{*} \phi^{a}=d \pi^{*} \phi^{a}+\epsilon_{b c}^{a} A^{b} \pi^{*} \phi^{c} .
$$

By standard results $[17,18]$, the covariant exterior derivative of $D_{A} \pi^{*} \phi$, given by

$$
d_{A} D_{A} \pi^{*} \phi^{a}=d D_{A} \pi^{*} \phi^{a}+\epsilon_{b c}^{a} A^{b} \wedge D_{A} \pi^{*} \phi^{c},
$$

can also be written in the form

$$
d_{A} D_{A} \pi^{*} \phi^{a}=\epsilon_{b c}^{a} F^{b} \pi^{*} \phi^{c},
$$

where the $F^{a}$ are the components of the $\mathfrak{s u}(2)$-part of the curvature form of the connection form $A$, defined by

$$
F^{0}=d A^{0} \quad, \quad F^{a}=d A^{a}+\frac{1}{2} \epsilon_{b c}^{a} A^{b} \wedge A^{c},
$$

\footnotetext{
${ }^{15}$ Note that under the isomorphism $\mathfrak{u}(2) \cong \mathbb{R}^{4}$ given by mapping the basis $\left\{\sigma_{a} / 2 i \mid a \in\{0,1,2,3\}\right\}$ of $\mathfrak{u}(2)$ to the standard basis of $\mathbb{R}^{4}$, where $\sigma_{0}$ is the unit matrix and $\sigma_{1}, \sigma_{2}, \sigma_{3}$ are the Pauli matrices as before, the commutator in $\mathfrak{u}(2)$ corresponds to that induced by the (associative) quaternion product in $\mathbb{R}^{4}$ whereas the invariant scalar product in $\mathfrak{u}(2)$ given by $(X, Y)=-2 \operatorname{tr}(X Y)$ corresponds to the standard scalar product in $\mathbb{R}^{4}$. With respect to this orthonormal basis, the (totally covariant) structure constants for $\mathfrak{u}(2)$ are again given by the components of the $\epsilon$-tensor, which are those of the standard volume form on $\mathbb{R}^{3}$ : all structure constants for which one of the indices takes the value 0 vanish.
} 
and there is a product rule relating the ordinary exterior derivative to the covariant exterior derivative which in the case of importance here reads

$$
\begin{aligned}
d\left(\epsilon_{a b c} \pi^{*} \phi^{a} D_{A}\right. & \left.\pi^{*} \phi^{b} \wedge D_{A} \pi^{*} \phi^{c}\right) \\
= & \epsilon_{a b c} D_{A} \pi^{*} \phi^{a} \wedge D_{A} \pi^{*} \phi^{b} \wedge D_{A} \pi^{*} \phi^{c} \\
& +\epsilon_{a b c} \pi^{*} \phi^{a} d_{A} D_{A} \pi^{*} \phi^{b} \wedge D_{A} \pi^{*} \phi^{c} \\
& \quad-\epsilon_{a b c} \pi^{*} \phi^{a} D_{A} \pi^{*} \phi^{b} \wedge d_{A} D_{A} \pi^{*} \phi^{c} .
\end{aligned}
$$

In fact, this expression vanishes identically since it represents an invariant horizontal 3form on $P$ which corresponds to a 3 -form on $M$, but there exists no non-zero 3 -form on a two-dimensional manifold. Now define

$$
\omega=\frac{1}{2} \epsilon_{a b c} A^{a} \wedge A^{b} \wedge D_{A} \pi^{*} \phi^{c}-\frac{1}{2} \epsilon_{a b c} \pi^{*} \phi^{a} A^{0} \wedge D_{A} \pi^{*} \phi^{b} \wedge D_{A} \pi^{*} \phi^{c} .
$$

Then using the above relations, together with the formula $\epsilon_{a b c} \epsilon^{a}{ }_{d e}=\delta_{b d} \delta_{c e}-\delta_{b e} \delta_{c d}$, we can calculate the exterior derivative of $\omega$ :

$$
\begin{aligned}
d \omega= & \frac{1}{2} \epsilon_{a b c} d A^{a} \wedge A^{b} \wedge D_{A} \pi^{*} \phi^{c}-\frac{1}{2} \epsilon_{a b c} A^{a} \wedge d A^{b} \wedge D_{A} \pi^{*} \phi^{c} \\
& +\frac{1}{2} \epsilon_{a b c} A^{a} \wedge A^{b} \wedge d_{A} D_{A} \pi^{*} \phi^{c}-\frac{1}{2} \epsilon_{a b c} \epsilon_{d e}^{c} A^{a} \wedge A^{b} \wedge A^{d} \wedge D_{A} \pi^{*} \phi^{e} \\
& -\frac{1}{2} \epsilon_{a b c} \pi^{*} \phi^{a} d A^{0} \wedge D_{A} \pi^{*} \phi^{b} \wedge D_{A} \pi^{*} \phi^{c} \\
& +\frac{1}{2} A^{0} \wedge d\left(\epsilon_{a b c} \pi^{*} \phi^{a} D_{A} \pi^{*} \phi^{b} \wedge D_{A} \pi^{*} \phi^{c}\right) \\
= & \frac{1}{2} \epsilon_{a b c} F^{a} \wedge A^{b} \wedge D_{A} \pi^{*} \phi^{c}-\frac{1}{2} \epsilon_{a b c} A^{a} \wedge F^{b} \wedge D_{A} \pi^{*} \phi^{c} \\
& -\frac{1}{4} \epsilon_{a b c} \epsilon_{d e}^{a} A^{d} \wedge A^{e} \wedge A^{b} \wedge D_{A} \pi^{*} \phi^{c}+\frac{1}{4} \epsilon_{a b c} \epsilon_{d e}^{b} A^{a} \wedge A^{d} \wedge A^{e} \wedge D_{A} \pi^{*} \phi^{c} \\
& +\frac{1}{2} \epsilon_{a b c} \epsilon_{d e}^{c} \pi^{*} \phi^{e} A^{a} \wedge A^{b} \wedge F^{d} \\
& -\frac{1}{2} \epsilon_{a b c} \epsilon_{d e}^{c} A^{a} \wedge A^{b} \wedge A^{d} \wedge D_{A} \pi^{*} \phi^{e} \\
& -\frac{1}{2} \epsilon_{a b c} \pi^{*} \phi^{a} F^{0} \wedge D_{A} \pi^{*} \phi^{b} \wedge D_{A} \pi^{*} \phi^{c} .
\end{aligned}
$$

The terms in the first and fifth line of the second equation vanish by the same argument of horizontality as before, while the terms in the second and fourth line vanish by symmetry. However, the term in the third line survives, i.e., we have

$$
d \omega=\delta_{a c} \delta_{b d} \pi^{*} \phi^{a} A^{b} \wedge A^{c} \wedge F^{d} .
$$

This means that $\omega$ is closed if (and also only if) the $\mathfrak{s u}(2)$-part of the curvature form $F$ vanishes, whereas there is no restriction on its $\mathfrak{u}(1)$-part. Moreover, it is easily verified that the image of $\pi^{*}(T M)$ under $D_{A} \pi^{*} \phi$ together with the (one-dimensional) orthogonal complement of $P \times \mathbb{R}^{3}$ in $P \times \mathbb{R}^{4}$, when transferred from $P \times \mathbb{R}^{4}$ to $V P$ by means of the canonical isomorphism that exists between the two since $P$ is a principal bundle, constitutes a three-dimensional invariant distribution $L$ on $P$ which is isotropic under $\omega$, so provided $\omega$ is closed, $\omega$ is multisymplectic and $L$ is the corresponding multilagrangian distribution. Of course, $L$ is not involutive. 
Remark 3 We emphasize again that the input data for the construction of the polysymplectic and multisymplectic forms in the last two examples allow for a natural interpretation in terms of Yang-Mills-Higgs field theory. Indeed, in the polysymplectic case, $A$ is an arbitrary $S U(2)$-connection and so we only have to make one assumption that is more restrictive than stated in Example 4. namely that the 1-form $e$ is (covariantly) holonomous, that is, of the form $e=D_{A} \phi$ where the Higgs field $\phi$ is a section of the adjoint bundle $P \times_{S U(2)} \mathbb{R}^{3}$. Moreover, since only the vertical part of $A$ appears in equation (95), it is clear that $\hat{\omega}$ is in fact completely independent of the choice of connection! In the multisymplectic case, we can argue that the relevant input data are (a) a principal $U(1)$-bundle $P_{0}$ over $M$ (or equivalently, a complex line bundle $L_{0}$ over $M$ with fixed hermitean fiber metric) and a given $U(1)$-connection form $A_{0}$ on $P_{0}$ (or equivalently, a given linear connection in $L_{0}$ preserving this fiber metric) and (b) a given embedding $\phi$ of $M$ into $\mathbb{R}^{3}$. Indeed, the principal $U(2)$-bundle $P$ over $M$ (corresponding to a rank 2 complex vector bundle $E_{0}$ over $M$ with fixed hermitean fiber metric) and the $U(2)$-connection form $A$ on $P$ (corresponding to a linear connection in $E_{0}$ preserving this fiber metric) that appear in Example 5 are then obtained by the process of extension of structure group, using the embedding homomorphism of $U(1)$ into $U(2)$ that takes a phase to that phase times the unit $(2 \times 2)$-matrix; moreover, this construction guarantees that the $\mathfrak{s u}(2)$-part of the curvature form $F$ will vanish. Conversely, if this condition is satisfied, then according to the Ambrose-Singer theorem, $P$ can be reduced to the holonomy bundle of $A$, whose structure group is the holonomy group of $A$ which has connected one-component $U(1)$ : this means that except for a possible discrete part, we are back to the previous situation. It should also be emphasized that from a purely topological point of view, reduction from $U(2)$ to $U(1)$ is always possible, e.g., over compact Riemann surfaces [20, Theorem 10 \& Corollary, p. 63], so the restriction made here really only concerns the connection form $A$, not the bundle $P$. Moreover, in this situation, the truncated adjoint bundle $P \times_{U(2)} \mathbb{R}^{3}$ will be trivial and so the Higgs field $\phi$ with its injective (covariant $=$ ordinary) derivative will provide an immersion of $M$ into $\mathbb{R}^{3}$ : it is only slightly more restrictive to assume that this immersion is in fact an embedding. Finally, an important observation that applies to both settings is that, as already mentioned, the polysymplectic form $\hat{\omega}$ of Example 4 and the multisymplectic form $\omega$ of Example 5 are both invariant under the action of the respective structure groups $S U(2)$ and $U(2)$ on the total space $P$ : expressed in physical language, this means that they are gauge invariant.

We expect it to be possible to apply similar procedures, with $S U(2)$ replaced by some compact three-dimensional Lie group which is solvable and $U(2)$ replaced by a compact four-dimensional Lie group which is a suitable one-dimensional extension thereof, to construct polysymplectic and multisymplectic fiber bundles $P$ where $P$ is still a compact manifold but $L$ is now involutive. However, in our view the real challenge is to come up with examples of polysymplectic and multisymplectic fiber bundles that appear naturally as multiphase spaces of physically realistic covariant hamiltonian field theories but, just as in the above cases, cannot be obtained by taking the affine dual of the first order jet bundle of some configuration bundle. This question is presently under investigation. 


\section{The Darboux Theorem}

Now we are able to prove the Darboux theorem for poly- and multilagrangian forms. Here, the specific algebraic structure of poly- and multilagrangian subspaces identified in the first two sections turns out to be crucial, in the sense that this central theorem can, in all cases, be proved by appropriately adapting the procedure used to prove the classical Darboux theorem for symplectic forms. (See, for instance, Ref. [19]).

Theorem 10 (Polylagrangian Darboux theorem) Let $P$ be a polylagrangian fiber bundle over an $n$-dimensional manifold $M$ with polylagrangian $(k+1)$-form $\hat{\omega}$ of rank $N$ taking values in a fixed $\hat{n}$-dimensional vector bundle $\hat{T}$ over the same manifold $M$ and with polylagrangian distribution $L$, which is assumed to be involutive (recall this is automatic if $\hat{n} \geqslant 3$ ), and let $\left\{\hat{e}_{a} \mid 1 \leqslant a \leqslant \hat{n}\right\}$ be a basis of local sections of $\hat{T}$. Then around any point of $P$ (within the domain of the given basis of local sections), there exists a system of local coordinates $\left(x^{\mu}, q^{i}, p_{i_{1} \ldots i_{k}}^{a}, r^{\kappa}\right)(1 \leqslant \mu \leqslant n, 1 \leqslant a \leqslant \hat{n}, 1 \leqslant i \leqslant N$, $1 \leqslant i_{1}<\ldots<i_{k} \leqslant N, 1 \leqslant \kappa \leqslant \operatorname{dim}$ ker $\left.\hat{\omega}\right)$, called Darboux coordinates or canonical coordinates, such that

$$
\hat{\omega}=\frac{1}{k !} d p_{i_{1} \ldots i_{k}}^{a} \wedge d q^{i_{1}} \wedge \ldots \wedge d q^{i_{k}} \otimes \hat{e}_{a},
$$

and such that (locally) $L$ is spanned by the vector fields $\partial / \partial p_{i_{1} \ldots i_{k}}^{a}$ and $\partial / \partial r^{\kappa}$ while ker $\hat{\omega}$ is spanned by the vector fields $\partial / \partial r^{\kappa}$. In the polysymplectic case this expansion takes the form

$$
\hat{\omega}=\left(d q^{i} \wedge d p_{i}^{a}\right) \otimes \hat{e}_{a}
$$

Similarly we have

Theorem 11 (Multilagrangian Darboux theorem) Let $P$ be a multilagrangian fiber bundle over an $n$-dimensional manifold $M$ with multilagrangian $(k+1)$-form $\omega$ of rank $N$ and horizontality degree $k+1-r$, where $1 \leqslant r \leqslant k$ and $k+1-r \leqslant n$, and with multilagrangian distribution $L$, which is assumed to be involutive (recall this is automatic if $\left.\left(\begin{array}{c}n \\ k+1-r\end{array}\right) \geqslant 3\right)$. Then around any point of $P$, there exists a system of local coordinates $\left(x^{\mu}, q^{i}, p_{i_{1} \ldots i_{s} ; \mu_{1} \ldots \mu_{k-s}}, r^{\kappa}\right)\left(0 \leqslant s \leqslant r-1,1 \leqslant \mu \leqslant n, 1 \leqslant i \leqslant N, 1 \leqslant i_{1}<\ldots<i_{s} \leqslant N\right.$, $1 \leqslant \mu_{1}<\ldots<\mu_{k-s} \leqslant N, 1 \leqslant \kappa \leqslant \operatorname{dim}$ ker $\left.\omega\right)$, called Darboux coordinates or canonical coordinates, such that

$$
\omega=\sum_{s=0}^{r-1} \frac{1}{s !} \frac{1}{(k-s) !} d p_{i_{1} \ldots i_{s} ; \mu_{1} \ldots \mu_{k-s}} \wedge d q^{i_{1}} \wedge \ldots \wedge d q^{i_{s}} \wedge d x^{\mu_{1}} \wedge \ldots \wedge d x^{\mu_{k-s}}
$$

and such that (locally) $L$ is spanned by the vector fields $\partial / \partial p_{i_{1} \ldots i_{s} ; \mu_{1} \ldots \mu_{k-s}}$ and $\partial / \partial r^{\kappa}$ while ker $\omega$ is spanned by the vector fields $\partial / \partial r^{\kappa}$. In these coordinates, its symbol is given by

$$
\hat{\omega}=\frac{1}{(r-1) !} \frac{1}{(k+1-r) !}\left(d p_{i_{1} \ldots i_{r-1} ; \mu_{1} \ldots \mu_{k+1-r}} \wedge d q^{i_{1}} \wedge \ldots \wedge d q^{i_{r-1}}\right) \otimes\left(d x^{\mu_{1}} \wedge \ldots \wedge d x^{\mu_{k+1-r}}\right) .
$$


In the multisymplectic case these expansions take the form

$$
\omega=d q^{i} \wedge d p_{i}^{\mu} \wedge d^{n} x_{\mu}-d p \wedge d^{n} x
$$

and

$$
\hat{\omega}=\left(d q^{i} \wedge d p_{i}^{\mu}\right) \otimes d^{n} x_{\mu}
$$

where

$$
d^{n} x_{\mu}=\mathrm{i}_{\partial_{\mu}} d^{n} x=\frac{1}{(n-1) !} \epsilon_{\mu \mu_{1} \ldots \mu_{n-1}} d x^{\mu_{1}} \wedge \ldots \wedge d x^{\mu_{n-1}}
$$

Proof: For the sake of simplicity, we concentrate on the multilagrangian case: the proof for the other case is entirely analogous, requiring only small and rather obvious modifications.

Due to the local character of this theorem and since the kernel of $\omega$, the multilagrangian subbundle $L$ and the vertical bundle $V P$ are all involutive, with $\operatorname{ker} \omega \subset L \subset V P$, we can without loss of generality work in a local chart of the manifold $P$ around the chosen reference point in which the corresponding foliations are "straightened out", so we may assume that $P \cong \mathbb{R}^{n} \oplus \mathbb{R}^{N} \oplus L_{0} \oplus K_{0}$ with $V P \cong \mathbb{R}^{N} \oplus L_{0} \oplus K_{0}, L \cong L_{0} \oplus K_{0}$ and ker $\omega \cong K_{0}$ with fixed subspaces $L_{0}$ and $K_{0}$ and such that the aforementioned reference point corresponds to the origin. We also take $\omega_{0}$ to be the constant multilagrangian form, with multilagrangian distribution $L$, obtained by spreading $\omega(0)$, the value of the multilagrangian form $\omega$ at the origin, all over the space $P$; then the existence of canonical coordinates for $\omega_{0}$, in the form given by equation (99), is already guaranteed by the algebraic Darboux theorem of the previous chapter (Theorem 6).

Now consider the family of $(k+1)$-forms given by $\omega_{t}=\omega_{0}+t\left(\omega-\omega_{0}\right)$, for every $t \in \mathbb{R}$. Obviously, $\omega_{t}(0)=\omega_{0}$ for every $t \in \mathbb{R}$, which is non-degenerate on $K_{0}^{\prime}=\mathbb{R}^{n} \oplus \mathbb{R}^{N} \oplus L_{0}$ (a complement of $K_{0}$ in $P$ ). Since non-degeneracy is an open condition, and using a compactness argument with respect to the parameter $t$, we conclude that there is an open neighborhood of 0 such that, for all $t$ satisfying $0 \leqslant t \leqslant 1$ and all points $p$ in this neighborhood, $\omega_{t}(p)$ is non-degenerate on $K_{0}^{\prime}=\mathbb{R}^{n} \oplus \mathbb{R}^{N} \oplus L_{0}$, that is, its kernel equals $K_{0}$. Moreover, for all $t$ satisfying $0 \leqslant t \leqslant 1$ and all points $p$ in this neighborhood, the subspace $L_{0}$, being isotropic for $\omega_{0}$ as well as for $\omega(p)$, is also isotropic for $\omega_{t}(p)$ and, since it contains the kernel of $\omega_{t}(p)$ and has the right dimension as given by equation (57), is even multilagrangian for $\omega_{t}(p)$, according to Proposition 5. On the other hand, we have $d \omega_{0}=0$ (trivially) and $d \omega=0$ (by hypothesis), so we can apply an appropriate version of the Poincaré lemma (see Appendix B) to prove, in some open neighborhood of the point 0 in $P$ (contained in the previous one), existence of a $k$-form $\alpha$ satisfying $d \alpha=\omega_{0}-\omega$ and $\alpha^{b}(L)=0$. Now take $X_{t}$ to be the unique time dependent vector field on $P$ taking values in $L_{0} 16$ defined by

$$
\mathrm{i}_{X_{t}} \omega_{t}=\alpha
$$

\footnotetext{
${ }^{16}$ It is at this point that we make essential use of the hypothesis that $L_{0}$ is multilagrangian and not just isotropic or even maximal isotropic (with respect to $\omega_{t}(p)$, in this case).
} 
Let $F_{t} \equiv F_{(0, t)}$ be its flux beginning at 0 , once again defined, for $0 \leqslant t \leqslant 1$, in some open neighborhood of the point 0 in $P$ (contained in the previous one). Then it follows that

$$
\begin{aligned}
\left.\frac{d}{d s}\right|_{s=t} F_{s}^{*} \omega_{s} & =F_{t}^{*}\left(\left.\frac{d}{d s}\right|_{s=t} \omega_{s}\right)+\left.\frac{d}{d s}\right|_{s=t} F_{s}^{*} \omega_{t} \\
& =F_{t}^{*}\left(\omega-\omega_{0}+L_{X_{t}} \omega_{t}\right) \\
& =F_{t}^{*}\left(\omega-\omega_{0}+d\left(\mathrm{i}_{X_{t}} \omega_{t}\right)\right) \\
& =F_{t}^{*}\left(\omega-\omega_{0}+d \alpha\right) \\
& =0
\end{aligned}
$$

Therefore, $F_{1}$ is the desired coordinate transformation, since $F_{1}^{*} \omega=F_{1}^{*} \omega_{1}=F_{0}^{*} \omega_{0}=\omega_{0}$.

\section{Conclusions and Outlook}

In this paper we have presented a concise definition of a new class of geometric structures which we propose to call polylagrangian or multilagrangian structures and which include as a special case the familiar polysymplectic or multisymplectic structures encountered in the hamiltonian formulation of classical field theory. All of them are defined in terms of differential forms satisfying an algebraic condition that amounts to postulating the existence of an isotropic subbundle which is "sufficiently large" - a condition which, when combined with the standard integrability conditions that the pertinent differential form should be closed and the aforementioned "sufficiently large" isotropic subbundle should be involutive (only in two space-time dimensions is this not automatic), allows to derive a Darboux theorem assuring the existence of "canonical" local coordinates around each point. Another characteristic feature of all these structures is that they are naturally defined on the total spaces of fiber bundles whose base space is interpreted as the spacetime manifold of field theory. Moreover, there is a standard class of examples defined by bundles of partially horizontal forms over the total space of another fiber bundle (the socalled configuration bundle), which includes the multiphase spaces of interest in physics and is the analogue of cotangent bundles of manifolds as a standard class of examples of symplectic manifolds. To our knowledge, this is the first example of a natural geometric structure with important physical applications that is defined by a differential form (or even a tensor field) of degree strictly larger than 2 and strictly smaller than the dimension of the underlying manifold.

Starting from this basis, there is a number of rather obvious questions that arise, most of which are closely interrelated. One of them concerns the structure of the underlying Lie group: interpreting these structures as $G$-structures, what is the nature of $G$ ? Other questions refer to the definition of Poisson brackets (see the discussion in Refs $[5-7,21]$ ), 
the definition of actions of Lie groups and, more generally, of Lie groupoids on polysymplectic/polylagrangian or multisymplectic/multilagrangian fiber bundles, the construction of a corresponding momentum map (which would provide a general framework for the construction of Noether currents and the energy-momentum tensor within a direct and manifestly covariant hamiltonian approach), the formulation of a Marsden-Weinstein reduction procedure and, last but by no means least, the explicit construction of other classes of examples, in particular, analogues of the coadjoint orbit construction of symplectic geometry. All these problems are completely open and certainly will be the subject of much research in the future.

\section{A Some Counterexamples}

In this appendix, we wish to provide additional evidence for the conceptual simplicity and usefulness of our definition of polylagrangian and multilagrangian structures by investigating, in a purely algebraic setting and for the simplest case of vector-valued 2-forms, various structural properties of polysymplectic forms that can be introduced directly for general vector-valued 2-forms. By constructing explicit counterexamples, we will show, however, that none of them is sufficiently strong to replace the requirement of existence of a polylagrangian subspace: this condition must therefore be imposed separately and is then sufficient to imply all the others, so that - in contrast to what is done, e.g., in Ref. [22] - we have refrained from including any of them into our definition of a polysymplectic structure.

Suppose, as in Section 1, that $V$ and $\hat{T}$ are finite-dimensional real vector spaces, with $\hat{n} \equiv \operatorname{dim} \hat{T}$, and assume that $\hat{\omega} \in \bigwedge^{2} V^{*} \otimes \hat{T}$ is an arbitrary $\hat{T}$-valued 2-form on $V$. Given any linear form $\hat{t}^{*} \in \hat{T}^{*}$ on $\hat{T}$, we consider the projection $\omega_{\hat{t}^{*}}=\left\langle\hat{t}^{*}, \hat{\omega}\right\rangle$ of $\hat{\omega}$ along $\hat{t}^{*}$ which is an ordinary 2 -form on $V$, and we define its rank to be equal to half the dimension of its support, which in turn can be defined as the annihilator of its kernel [23]:17

$$
\operatorname{rk}\left(\omega_{\hat{t}^{*}}\right)=\frac{1}{2} \operatorname{dim} \operatorname{supp} \omega_{\hat{t}^{*}}=\frac{1}{2}\left(\operatorname{dim} V-\operatorname{dim} \operatorname{ker} \omega_{\hat{t}^{*}}\right) .
$$

Now note that the linear mapping

$$
\begin{aligned}
& \hat{T}^{*} \longrightarrow \bigwedge^{2} V^{*} \\
& \hat{t}^{*} \longmapsto \omega_{\hat{t}^{*}}
\end{aligned}
$$

induces, for every integer $k \geqslant 1$, a canonically defined linear mapping

$$
\begin{array}{cc}
\bigvee^{k} \hat{T}^{*} & \longrightarrow \bigwedge^{2 k} V^{*} \\
P & \longmapsto P(\hat{\omega})
\end{array}
$$

where we have identified the space $\bigvee^{k} \hat{T}^{*}$ of covariant symmetric tensors of degree $k$ over $\hat{T}$ with the space of homogeneous polynomials $P$ of degree $k$ on $\hat{T}$. Explicitly, in

\footnotetext{
${ }^{17}$ Thus our definition of rank differs from that Ref. [23] by a factor of 2 .
} 
terms of a basis $\left\{\hat{e}_{a} \mid 1 \leqslant a \leqslant \hat{n}\right\}$ of $\hat{T}$, with dual basis $\left\{\hat{e}^{a} \mid 1 \leqslant a \leqslant \hat{n}\right\}$ of $\hat{T}^{*}$, we write $\omega^{a}=\omega_{\hat{e}^{a}}=\left\langle\hat{e}^{a}, \hat{\omega}\right\rangle(1 \leqslant a \leqslant \hat{n})$ and obtain

$$
P=P_{a_{1} \ldots a_{k}} \hat{e}^{a_{1}} \vee \ldots \vee \hat{e}^{a_{k}} \Longrightarrow P(\hat{\omega})=P_{a_{1} \ldots a_{k}} \omega^{a_{1}} \wedge \ldots \wedge \omega^{a_{k}} .
$$

This allows us to introduce the following terminology:

Definition 6 Let $V$ and $\hat{T}$ be finite-dimensional vector spaces and let $\hat{\omega}$ be a $\hat{T}$-valued 2 -form on $V$. We say that $\hat{\omega}$ has constant rank $N$ if $\operatorname{rk}\left(\omega_{\hat{t}^{*}}\right)=N$ for every $\hat{t}^{*} \in \hat{T}^{*} \backslash\{0\}$ and that $\hat{\omega}$ has uniform rank $N$ if the linear mapping (106) is injective for $k=N$ and identically zero for $k=N+1$.

Using multi-indices $\alpha=\left(\alpha_{1}, \ldots, \alpha_{\hat{n}}\right) \in \mathbb{N}^{\hat{n}}$, we set

$$
\hat{e}^{\alpha}=\left(\hat{e}^{1}\right)^{\alpha_{1}} \vee \ldots \vee\left(\hat{e}^{\hat{n}}\right)^{\alpha_{\hat{n}}} \quad \text { where } \quad\left(\hat{e}^{a}\right)^{\alpha_{a}}=\hat{e}^{a} \vee \ldots \vee \hat{e}^{a} \quad\left(\alpha_{a} \text { times }\right)
$$

and

$$
\omega^{\alpha}=\left(\omega^{1}\right)^{\alpha_{1}} \wedge \ldots \wedge\left(\omega^{\hat{n}}\right)^{\alpha_{\hat{n}}} \quad \text { where } \quad\left(\omega^{a}\right)^{\alpha_{a}}=\omega^{a} \wedge \ldots \wedge \omega^{a} \quad\left(\alpha_{a} \text { times }\right)
$$

to rewrite equation (107) in the form

$$
P=\sum_{|\alpha|=k} P_{\alpha} \hat{e}^{\alpha} \Longrightarrow P(\hat{\omega})=\sum_{|\alpha|=k} P_{\alpha} \omega^{\alpha}
$$

Since $\left\{\hat{e}^{\alpha}|| \alpha \mid=k\right\}$ is a basis of $\bigvee^{k} \hat{T}^{*}$, requiring $\hat{\omega}$ to have uniform rank $N$ amounts to imposing the following conditions:

$$
\begin{gathered}
\left\{\omega^{\alpha}|| \alpha \mid=N\right\} \text { is linearly independent } \\
\omega^{\alpha}=0 \text { for }|\alpha|=N+1
\end{gathered}
$$

It is in this form that the requirement of uniform rank appears in the definition of a polysymplectic form adopted in Ref. [22].

To gain a better understanding for the conditions of constant rank and of uniform rank introduced above, we note first of all that they both generalize the standard notion of rank for ordinary forms. Indeed, when $\hat{n}=1$, that is, given an ordinary 2-form $\omega$ of rank $N$ on $V$, we can choose a canonical basis $\left\{e_{1}, \ldots, e_{N}, f^{1}, \ldots, f^{N}\right\}$ of a subspace of $V$ complementary to the kernel of $\omega$, with dual basis $\left\{e^{1}, \ldots, e^{N}, f_{1}, \ldots, f_{N}\right\}$ of the subspace $\operatorname{supp} \omega$ of $V^{*}$, to conclude that $\omega=e^{i} \wedge f_{i}$ and therefore

$$
\omega^{N}= \pm e^{1} \wedge \ldots \wedge e^{N} \wedge f_{1} \wedge \ldots \wedge f_{N} \neq 0, \quad \omega^{N+1}=0 .
$$

In other words, the rank of $\omega$ can be characterized as that positive integer $N$ for which $\omega^{N} \neq 0$ but $\omega^{N+1}=0$. From this observation, it follows that, in the general case considered before, the requirement of uniform rank implies that of constant rank because it guarantees that for every $\hat{t}^{*} \in \hat{T}^{*} \backslash\{0\}$, we have $\omega_{\hat{t}^{*}}^{N} \neq 0$ and $\omega_{\hat{t}^{*}}^{N+1}=0$, that is, $\operatorname{rk}\left(\omega_{\hat{t}^{*}}\right)=N$. However, the converse does not hold, as shown by the following 
Example $6 \quad(\hat{n}=2, N=2, \operatorname{dim} V=4, \operatorname{ker} \hat{\omega}=\{0\})$ :

Let $V=\mathbb{R}^{4}, \hat{T}=\mathbb{R}^{2}$ and consider the $\mathbb{R}^{2}$-valued 2-form $\hat{\omega}$ built from the following two ordinary 2-forms:

$$
\omega^{1}=d x \wedge d y+d u \wedge d v \quad, \quad \omega^{2}=d x \wedge d u-d y \wedge d v .
$$

Then for $\hat{t}^{*}=\hat{t}_{a}^{*} \hat{e}^{a} \in\left(\mathbb{R}^{2}\right)^{*}$, we have

$$
\omega_{\hat{t}^{*}}=\hat{t}_{a}^{*} \omega^{a}=d x \wedge\left(\hat{t}_{1}^{*} d y+\hat{t}_{2}^{*} d u\right)+d v \wedge\left(\hat{t}_{2}^{*} d y-\hat{t}_{1}^{*} d u\right) .
$$

Thus we obtain, for every $\hat{t}^{*} \neq 0$,

$$
\left(\omega_{\hat{t}^{*}}\right)^{2} \equiv \omega_{\hat{t}^{*}} \wedge \omega_{\hat{t}^{*}}=\left(\left(\hat{t}_{1}^{*}\right)^{2}+\left(\hat{t}_{2}^{*}\right)^{2}\right) d x \wedge d y \wedge d u \wedge d v \neq 0
$$

whereas, due to the fact that we are in a four-dimensional space,

$$
\left(\omega_{\hat{t}^{*}}\right)^{3} \equiv \omega_{\hat{t}^{*}} \wedge \omega_{\hat{t}^{*}} \wedge \omega_{\hat{t}^{*}}=0
$$

which guarantees that $\hat{\omega}$ has constant rank 2 . However, $\hat{\omega}$ does not have uniform rank 2 , since

$$
\omega^{1} \wedge \omega^{2}=0
$$

On the other hand, polysymplectic forms do have uniform rank:

Proposition 6 Let $V$ and $\hat{T}$ be finite-dimensional vector spaces and let $\hat{\omega}$ be a $\hat{T}$-valued polysymplectic form of rank $N$ on $V$. Then $\hat{\omega}$ has uniform rank $N$.

Proof: Introducing a (polysymplectic) canonical basis in which

$$
\hat{\omega}=\left(e_{i}^{a} \wedge e^{i}\right) \otimes \hat{e}_{a}
$$

or equivalently

$$
\omega^{a}=e_{i}^{a} \wedge e^{i} \quad(1 \leqslant a \leqslant \hat{n})
$$

suppose that $\alpha=\left(\alpha_{1}, \ldots, \alpha_{\hat{n}}\right) \in \mathbb{N}^{\hat{n}}$ is a multi-index of degree $k$ (i.e., such that $|\alpha|=$ $\left.\alpha_{1}+\ldots+\alpha_{\hat{n}}=k\right)$ and consider the form

$$
\begin{aligned}
\omega^{\alpha}= \pm & \left(\left(e^{i_{1}^{1}} \wedge \ldots \wedge e^{i_{\alpha_{1}}^{1}}\right) \wedge \ldots \wedge\left(e^{i_{1}^{\hat{n}}} \wedge \ldots \wedge e^{i_{\alpha_{\hat{n}}}^{\hat{n}}}\right)\right) \\
& \wedge\left(e_{i_{1}^{1}}^{1} \wedge \ldots \wedge e_{i_{\alpha_{1}}^{1}}^{1}\right) \wedge \ldots \wedge\left(e_{i_{1}^{\hat{n}}}^{\hat{n}} \wedge \wedge e_{i_{\alpha_{\hat{n}}}^{\hat{n}}}^{\hat{n}}\right) .
\end{aligned}
$$

Obviously any such form vanishes when $k=N+1$ since it then contains an exterior product of $(N+1) 1$-forms $e^{i}$ belonging to an $N$-dimensional subspace. On the other hand, all these forms are linearly independent when $k=N$ since $\omega^{\alpha}$ then contains the exterior product $e^{1} \wedge \ldots \wedge e^{N}$ multiplied by the exterior product of $\alpha_{1}$ 1-forms of type $e_{i}^{1}$ with $\ldots$ with $\alpha_{\hat{n}} 1$-forms of type $e_{i}^{\hat{n}}$; thus $\omega^{\alpha}$ and $\omega^{\beta}$ belong to different subspaces of $\bigwedge^{2 N} V^{*}$ whenever $\alpha \neq \beta$. 
The converse statement, as we shall see shortly, is remote from being true. In fact, if it were true, then if $\hat{n} \geqslant 2$, it should be possible to construct the polylagrangian subspace as the sum of the kernels of the projected forms, as required by Theorem 11. Therefore, it should be possible to show that the subspace defined as the sum of these kernels is isotropic. And indeed, as a partial result in this direction, we have the following

Proposition 7 Let $V$ and $\hat{T}$ be finite-dimensional vector spaces and let $\hat{\omega}$ be a $\hat{T}$ valued 2-form of uniform rank $N$ on $V$. Then for any $\hat{t}_{1}^{*}, \hat{t}_{2}^{*} \in \hat{T}^{*} \backslash\{0\}$, the kernel of $\omega_{\hat{t}_{1}^{*}}$ is isotropic with respect to $\omega_{\hat{t}_{2}^{*}}$.

Proof: $\quad$ Given $u, v \in \operatorname{ker} \omega_{\hat{t}_{1}^{*}}$, we have

$$
\mathrm{i}_{u} \omega_{\hat{t}_{1}^{*}}^{N}=N \mathrm{i}_{u} \omega_{\hat{t}_{1}^{*}} \wedge \omega_{\hat{t}_{1}^{*}}^{N-1}=0 \quad, \quad \mathrm{i}_{v} \omega_{\hat{t}_{1}^{*}}^{N}=N \mathrm{i}_{v} \omega_{\hat{t}_{1}^{*}} \wedge \omega_{\hat{t}_{1}^{*}}^{N-1}=0
$$

and therefore

$$
\omega_{\hat{t}_{2}^{*}}(u, v) \omega_{\hat{t}_{1}^{*}}^{N}=\mathrm{i}_{u} \mathrm{i}_{v}\left(\omega_{\hat{t}_{2}^{*}} \wedge \omega_{\hat{t}_{1}^{*}}^{N}\right)=0 .
$$

Using that $\omega_{\hat{t}_{1}^{*}}^{N} \neq 0$, it follows that $\omega_{\hat{t}_{2}^{*}}(u, v)=0$.

However, isotropy of the subspace defined as the sum of the kernels of all the projected forms, which is equivalent to the (stronger) condition that for any $\hat{t}_{1}^{*}, \hat{t}_{2}^{*}, \hat{t}_{3}^{*} \in \hat{T}^{*} \backslash\{0\}$, ker $\omega_{\hat{t}_{1}^{*}}$ and ker $\omega_{\hat{t}_{2}^{*}}$ are orthogonal under $\omega_{\hat{t}_{3}^{*}}$, i.e., that

$$
\omega_{\hat{t}_{3}^{*}}\left(u_{1}, u_{2}\right)=0 \quad \text { for } u_{1} \in \operatorname{ker} \omega_{\hat{t}_{1}^{*}} \text { and } u_{2} \in \operatorname{ker} \omega_{\hat{t}_{2}^{*}} \text {, }
$$

cannot be derived from the condition of uniform rank. A nice counterexample is obtained by choosing $V$ and $\hat{T}$ to be the same space, supposing it to be a Lie algebra $\mathfrak{g}$ and defining $\hat{\omega}$ to be the commutator in $\mathfrak{g}$. Then for $\hat{t}^{*} \in \mathfrak{g}^{*}$, the kernel $\operatorname{ker} \omega_{\hat{t}^{*}}$ and the support $\operatorname{supp} \omega_{\hat{t}^{*}}$ of the projected form $\omega_{\hat{t}^{*}}$ are the isotropy algebra of $\hat{t}^{*}$ and the tangent space to the coadjoint orbit passing through $\hat{t}^{*}$, respectively. There is one and only one semisimple Lie algebra for which $\hat{\omega}$ has constant rank, since this condition states that all coadjoint orbits except the trivial one, $\{0\}$, should have the same dimension: this is the algebra of type $A_{1}$, that is, $\mathbb{R}^{3}$ equipped with the vector product $\times$.

Example $7 \quad(\hat{n}=3, N=1, \operatorname{dim} V=3, \operatorname{ker} \hat{\omega}=\{0\})$ :

Let $V=\hat{T}=\mathbb{R}^{3}$ and consider the $\mathbb{R}^{3}$-valued 2-form $\hat{\omega}$ built from the following three ordinary 2-forms:

$$
\omega^{1}=d y \wedge d z \quad, \quad \omega^{2}=d z \wedge d x \quad, \quad \omega^{3}=d x \wedge d y .
$$

Then for $\hat{t}^{*}=\hat{t}_{a}^{*} \hat{e}^{a} \in\left(\mathbb{R}^{3}\right)^{*}$, we have

$$
\omega_{\hat{t}^{*}}=\hat{t}_{a}^{*} \omega^{a}=\hat{t}_{1}^{*} d y \wedge d z+\hat{t}_{2}^{*} d z \wedge d x+\hat{t}_{3}^{*} d x \wedge d y .
$$


Obviously, $\omega^{1}, \omega^{2}$ and $\omega^{3}$ are linearly independent and hence $\hat{\omega}$ has uniform rank 1 , since there exists no non-zero 4-form on a three-dimensional space. On the other hand, we have

$$
\operatorname{ker} \omega_{\hat{t}^{*}}=\left\langle\hat{t}_{1}^{*} \frac{\partial}{\partial x}+\hat{t}_{2}^{*} \frac{\partial}{\partial y}+\hat{t}_{3}^{*} \frac{\partial}{\partial z}\right\rangle
$$

Therefore, the intersection of the three kernels ker $\omega^{1}$, ker $\omega^{2}$ and $\operatorname{ker} \omega^{3}$ is $\{0\}$ (i.e., $\hat{\omega}$ is non-degenerate). However, ker $\omega^{1}$ and ker $\omega^{2}$ are orthogonal under $\omega^{1}$ and under $\omega^{2}$ but not under $\omega^{3}$. Now if there existed a polylagrangian subspace it would have to coincide with the sum of the kernels of all the projected forms, but that is the whole space $\mathbb{R}^{3}$, which is not isotropic. Thus $\hat{\omega}$ does not admit a polylagrangian subspace.

Finally, we observe that even if the sum of the kernels of all the projected forms is an isotropic subspace with respect to $\hat{\omega}$, it may still fail to be a polylagrangian subspace, as shown by the following

Example $8 \quad(\hat{n}=2, N=2, \operatorname{dim} V=5, \operatorname{ker} \hat{\omega}=\{0\})$ :

Let $V=\mathbb{R}^{5}, \hat{T}=\mathbb{R}^{2}$ and consider the $\mathbb{R}^{2}$-valued 2-form $\hat{\omega}$ built from the following two ordinary 2-forms:

$$
\omega^{1}=d x^{1} \wedge d x^{4}+d x^{2} \wedge d x^{3} \quad, \quad \omega^{2}=d x^{1} \wedge d x^{3}-d x^{2} \wedge d x^{5} .
$$

Then for $\hat{t}^{*}=\hat{t}_{a}^{*} \hat{e}^{a} \in\left(\mathbb{R}^{2}\right)^{*}$, we have

$$
\omega_{\hat{t}^{*}}=\hat{t}_{a}^{*} \omega^{a}=d x^{1} \wedge\left(\hat{t}_{1}^{*} d x^{4}+\hat{t}_{2}^{*} d x^{3}\right)+d x^{2} \wedge\left(\hat{t}_{1}^{*} d x^{3}-\hat{t}_{2}^{*} d x^{5}\right) .
$$

Obviously, $\omega^{1}, \omega^{2}$ and the forms

$$
\begin{gathered}
\left(\omega^{1}\right)^{2} \equiv \omega^{1} \wedge \omega^{1}=2 d x^{1} \wedge d x^{2} \wedge d x^{3} \wedge d x^{4}, \\
\omega^{1} \wedge \omega^{2}=d x^{1} \wedge d x^{2} \wedge d x^{4} \wedge d x^{5} \\
\left(\omega^{2}\right)^{2} \equiv \omega^{2} \wedge \omega^{2}=2 d x^{1} \wedge d x^{2} \wedge d x^{3} \wedge d x^{5}
\end{gathered}
$$

are linearly independent and hence $\hat{\omega}$ has uniform rank 2 , since there exists no non-zero 6 -form on a five-dimensional space. On the other hand, we have

$$
\operatorname{ker} \omega_{\hat{t}^{*}}=\left\langle\hat{t}_{1}^{*} \hat{t}_{2}^{*} \frac{\partial}{\partial x^{3}}-\left(\hat{t}_{2}^{*}\right)^{2} \frac{\partial}{\partial x^{4}}+\left(\hat{t}_{1}^{*}\right)^{2} \frac{\partial}{\partial x^{5}}\right\rangle \text {. }
$$

The intersection of the two kernels $\operatorname{ker} \omega^{1}$ and $\operatorname{ker} \omega^{2}$ is $\{0\}$ (i.e., $\hat{\omega}$ is non-degenerate). Note that their (direct) sum is the two-dimensional subspace of $V$, say $L^{\prime}$, spanned by $\partial / \partial x^{4}$ and $\partial / \partial x^{5}$, whereas the subspace spanned by all the kernels $\operatorname{ker} \omega_{\hat{t}^{*}}\left(\hat{t}^{*} \in \hat{T}^{*} \backslash\{0\}\right)$ is the three-dimensional subspace of $V$, say $L^{\prime \prime}$, spanned by $\partial / \partial x^{i}$ with $i=3,4,5$, and this is isotropic with respect to all the forms $\omega_{\hat{t}^{*}}\left(\hat{t}^{*} \in \hat{T}^{*} \backslash\{0\}\right)$. More than that: since its codimension is 2 , it is maximal isotropic with respect to all the forms $\omega_{\hat{t}^{*}}\left(\hat{t}^{*} \in \hat{T}^{*} \backslash\{0\}\right)$. Now if there existed a polylagrangian subspace it would have to coincide with $L^{\prime}$ and also with $L^{\prime \prime}$, but these two are not equal and do not have the right dimension, which according to equation (28) would have to be 4 : both of them are too small. Thus $\hat{\omega}$ does not admit a polylagrangian subspace. 
To summarize, the examples given above show that the hypothesis of existence of a polylagrangian subspace is highly non-trivial and very restrictive: as it seems, it cannot be replaced by any other hypothesis that is not obviously equivalent. The examples also show the great variety of possibilities for the "relative positions" of the kernels of the various projected forms that prevails when such a subspace does not exist. In this sense, the definition adopted in Ref. [22] is quite inconvenient, since it makes no reference to this subspace, thus hiding the central aspect of the theory.

To conclude, we want to add some remarks about the relation between the polylagrangian subspace, when it exists, and the more general class of maximal isotropic subspaces. First, we emphasize that in contrast with a polylagrangian subspace, maximal isotropic subspaces always exist. To prove this, it suffices to start out from an arbitrary one-dimensional subspace $L_{1}$, which is automatically isotropic, and construct a chain $L_{1} \subset L_{2} \subset \ldots$ of subspaces where $L_{p+1}$ is defined as the direct sum of $L_{p}$ and the onedimensional subspace spanned by some non-zero vector in its 1-orthogonal complement $L_{p}^{\hat{\omega}, 1}$. For dimensional reasons, this process must stop at some point, which means that at this point we have succeeded in constructing a maximal isotropic subspace. However, nothing guarantees that maximal isotropic subspaces resulting from different chains must have the same dimension, nor that there must exist some chain leading to a maximal isotropic subspace of sufficiently high dimension to be polylagrangian: this happens only in the special case of ordinary forms $(\hat{n}=1)$, where all maximal isotropic subspaces have the same dimension and where the notions of a polylagrangian subspace (or simply lagrangian subspace, in this case) and of a maximal isotropic subspace coincide.

Another important point concerns the relation between the notions of isotropic subspace and maximal isotropic subspace with respect to the form $\hat{\omega}$ and with respect to its projections. First, it is obvious that a subspace of $V$ is isotropic with respect to $\hat{\omega}$ if and only if it is isotropic with respect to each of the projected forms $\omega_{\hat{t}^{*}}\left(\hat{t}^{*} \in T^{*} \backslash\{0\}\right)$ or $\omega^{a}$ $(1 \leqslant a \leqslant \hat{n})$. However, this no longer holds when we substitute the term "isotropic" by the term "maximal isotropic": a subspace of $V$ that is maximal isotropic with respect to each of the projections of $\hat{\omega}$ is also maximal isotropic with respect to $\hat{\omega}$, but conversely, it can very well be maximal isotropic with respect to $\hat{\omega}$ (and hence isotropic with respect to each of the projections of $\hat{\omega}$ ) but fail to be maximal isotropic with respect to some of them. And finally, a polylagrangian subspace of $V$ is maximal isotropic with respect to each of the projections of $\hat{\omega}$ (this follows from Theorem 1), but as we have seen in the last example above, the converse is false: a subspace can be maximal isotropic with respect to each of the projections of $\hat{\omega}$ without being polylagrangian. All these statements illustrate the special nature of the polylagrangian subspace, already in the case of vector-valued 2 -forms. 


\section{B Poincaré Lemma}

In this appendix we want to state the Poincaré lemma in the form in which it is used in the proof of the Darboux theorem in Section 6.

Theorem 12 Let $\omega \in \Omega^{k}(P, \hat{T})$ be a closed form on a manifold $P$ taking values in a fixed vector space $\hat{T}$ and let $L$ be an involutive distribution on $P$. Suppose that $\omega$ is $(k-r)$ horizontal (with respect to $L$ ), i.e., such that for any $p \in P$ and all $v_{1}, \ldots, v_{r+1} \in L_{p}$, we have

$$
\mathrm{i}_{v_{1}} \ldots \mathrm{i}_{v_{r+1}} \omega_{p}=0 .
$$

Then for any point of $P$ there exist an open neighborhood $U$ of that point and a $(k-1)$ form $\theta \in \Omega^{k-1}(U, \hat{T})$ on $U$ which is also $(k-r)$-horizontal (with respect to $\left.L\right)$, i.e., such that for any $p \in U$ and all $v_{1}, \ldots, v_{r} \in L_{p}$, we have

$$
\mathrm{i}_{v_{1}} \ldots \mathrm{i}_{v_{r}} \theta_{p}=0
$$

and such that $\omega=d \theta$ on $U$.

Proof: Due to the local character of this theorem and since the subbundle $L$ of TP is involutive, we can without loss of generality work in a local chart of the manifold $P$ around the chosen reference point in which the foliation defined by $L$ is "straightened out", so we may assume that $P \cong K_{0} \oplus L_{0}$ with $L \cong L_{0}$ with fixed subspaces $K_{0}$ and $L_{0}$ and such that the aforementioned reference point corresponds to the origin. (In what follows, we shall omit the index 0 .) We also suppose that $\hat{T}=\mathbb{R}$, since we may prove the theorem separately for each component of $\omega$ and $\theta$, with respect to some fixed basis of $\hat{T}$.

For $t \in \mathbb{R}$, define the " $K$-contraction" $F_{t}^{K}: P \rightarrow P$ and the " $L$-contraction" $F_{t}^{L}: P \rightarrow P$ by $F_{t}^{K}(x, y)=(t x, y)$ and $F_{t}^{L}(x, y)=(x, t y)$; obviously, $F_{t}^{K}$ and $F_{t}^{L}$ are diffeomorphisms if $t \neq 0$ and are projections if $t=0$. Associated with each of these families of mappings there is a time dependent vector field which generates it in the sense that, for $t \neq 0$,

$$
X_{t}^{K}\left(F_{t}^{K}(x, y)\right)=\left.\frac{d}{d s} F_{s}^{K}(x, y)\right|_{s=t} \quad \text { and } \quad X_{t}^{L}\left(F_{t}^{L}(x, y)\right)=\left.\frac{d}{d s} F_{s}^{L}(x, y)\right|_{s=t} .
$$

Explicitly, for $t \neq 0$,

$$
X_{t}^{K}(x, y)=t^{-1}(x, 0) \quad \text { and } \quad X_{t}^{L}(x, y)=t^{-1}(0, y) .
$$

Define $\omega_{0}=\left(F_{0}^{L}\right)^{*} \omega$ and, for $\epsilon>0$,

$$
\theta_{\epsilon}=\int_{\epsilon}^{1} d t\left(\left(F_{t}^{L}\right)^{*}\left(\mathrm{i}_{X_{t}^{L}} \omega\right)+\left(F_{t}^{K}\right)^{*}\left(\mathrm{i}_{X_{t}^{K}} \omega_{0}\right)\right)
$$

as well as

$$
\theta=\lim _{\epsilon \rightarrow 0} \theta_{\epsilon}=\int_{0}^{1} d t\left(\left(F_{t}^{L}\right)^{*}\left(\mathrm{i}_{X_{t}^{L}} \omega\right)+\left(F_{t}^{K}\right)^{*}\left(\mathrm{i}_{X_{t}^{K}} \omega_{0}\right)\right) .
$$


To see that the $(k-1)$-forms $\theta$ and $\theta_{\epsilon}$ are well defined, consider $k-1$ vectors $\left(u_{i}, v_{i}\right) \in K \oplus L$ $(1 \leqslant i \leqslant k-1)$ and observe that, for $t \neq 0$,

$$
\left(F_{t}^{L}\right)^{*}\left(i_{X_{t}^{L}} \omega\right)_{(x, y)}\left(\left(u_{1}, v_{1}\right), \ldots,\left(u_{k-1}, v_{k-1}\right)\right)=\omega_{(x, t y)}\left((0, y),\left(u_{1}, t v_{1}\right), \ldots,\left(u_{k-1}, t v_{k-1}\right)\right)
$$

and

$$
\left(F_{t}^{K}\right)^{*}\left(i_{X_{t}^{K}} \omega_{0}\right)_{(x, y)}\left(\left(u_{1}, v_{1}\right), \ldots,\left(u_{k-1}, v_{k-1}\right)\right)=t^{k-1} \omega_{(t x, 0)}\left((x, 0),\left(u_{1}, 0\right), \ldots,\left(u_{k-1}, 0\right)\right) .
$$

Here we see easily that both expressions are differentiable in $t$ and provide $(k-1)$-forms which are $(k-r)$-horizontal and $(k-1)$-horizontal with respect to $L$, respectively. Thus, $\theta_{\epsilon}$ and $\theta$ are $(k-1)$-forms which are $(k-r)$-horizontal with respect to $L$. Moreover, since $d \omega=0$ and $d \omega_{0}=0$,

$$
\begin{aligned}
d \theta_{\epsilon} & =\int_{\epsilon}^{1} d t\left(\left(F_{t}^{L}\right)^{*}\left(\mathrm{i}_{X_{t}^{L}} \omega\right)+\left(F_{t}^{K}\right)^{*}\left(\mathrm{i}_{X_{t}^{K}} \omega_{0}\right)\right) \\
& =\int_{\epsilon}^{1} d t\left(\left(F_{t}^{L}\right)^{*}\left(L_{X_{t}^{L}} \omega\right)+\left(F_{t}^{K}\right)^{*}\left(L_{X_{t}^{K}} \omega_{0}\right)\right) \\
& =\int_{\epsilon}^{1} d t\left(\frac{d}{d t}\left(\left(F_{t}^{L}\right)^{*} \omega\right)+\frac{d}{d t}\left(\left(F_{t}^{K}\right)^{*} \omega_{0}\right)\right) \\
& =\omega-\left(F_{\epsilon}^{L}\right)^{*} \omega+\omega_{0}-\left(F_{\epsilon}^{K}\right)^{*} \omega_{0} .
\end{aligned}
$$

Taking the limit $\epsilon \rightarrow 0$, we get $\left(F_{\epsilon}^{L}\right)^{*} \omega \rightarrow \omega_{0}$ and $\left(F_{\epsilon}^{K}\right)^{*} \omega_{0} \rightarrow 0$ and hence $d \theta=\omega$.

\section{References}

[1] Th. De Donder: Théorie Invariante du Calcul des Variations, Gauthier-Villars, Paris 1935.

[2] H. Weyl: Geodesic Fields in the Calculus of Variations for Multiple Integrals, Ann. Math. 36 (1935) 607-629.

[3] M.J. Gotay, J. Isenberg, J.E. Marsden \& R. Montgomery: Momentum Maps and Classical Relativistic Fields. Part I: Covariant Field Theory, arXiv: physics/9801019v2.

[4] J.F. Cariñena, M. Crampin \& L.A. Ibort: On the Multisymplectic Formalism for First Order Field Theories, Diff. Geom. App. 1 (1991) 345-374.

[5] M. Forger \& H. Römer: A Poisson Bracket on Multisymplectic Phase Space, Rep. Math. Phys. 48 (2001) 211-218.

[6] M. Forger, C. Paufler \& H. Römer: The Poisson Bracket for Poisson Forms in Multisymplectic Field Theory, Rev. Math. Phys. 15 (2003) 705-743. 
[7] M. Forger, C. Paufler \& H. Römer: Hamiltonian Multivector Fields and Poisson Forms in Multisymplectic Field Theory, J. Math. Phys. 46 (2005) 112903, 29 pp.

[8] G. Martin: A Darboux Theorem for Multisymplectic Manifolds, Lett. Math. Phys. 16 (1988) 133-138.

[9] F. Cantrijn, L.A. Ibort \& M. De León: On the Geometry of Multisymplectic Manifolds, J. Austral. Math. Soc. (Series A) 66 (1999) 303-330.

[10] M. De León, D. De Diego e Santamaría-Merino: Tulczyjew's Triples and Lagrangian Submanifolds in Classical Field Theories, in: W. Sarlet \& F. Cantrjin (eds): Applied Differential Geometry and Mechanics, Univ. of Gent, Gent, Academic Press (2003) 21-47; arXiv: math-ph/03020026.

[11] J. Kijowski: A Finite-dimensional Canonical Formalism in the Classical Field Theory, Commun. Math. Phys. 30 (1973) 99-128; Multiphase Spaces and Gauge in Calculus of Variations, Bull. Acad. Pol. Sci. SMAP 22 (1974) 1219-1225.

[12] J. KiJowski \& W. Szczyrba: Multisymplectic Manifolds and the Geometrical Construction of the Poisson Brackets in the Classical Field Theory, in: "Géometrie Symplectique et Physique Mathématique", pp. 347-379, ed.: J.-M. Souriau, C.N.R.S., Paris 1975.

[13] J. Kijowski \& W. Szczyrba: Canonical Structure for Classical Field Theories, Commun. Math. Phys. 46 (1976) 183-206.

[14] C. Günther: The Polysymplectic Hamiltonian Formalism in Field Theory and Calculus of Variations I: the Local Case, J. Diff. Geom. 25 (1987) 23-53.

[15] A. Awane: k-symplectic structures, J. Math. Phys. 33 (1992) 4046-4052.

[16] L.G. Gomes: Polysymplectic and Multisymplectic Structures on Manifolds and Fiber Bundles, PhD thesis, Institute for Mathematics and Statistics, University of São

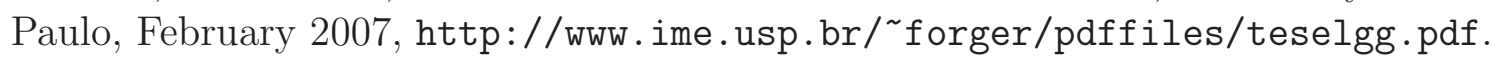

[17] W.H. Greub, S. Halperin \& R. Vanstone: Connections, Curvature and Cohomology, Academic Press, New York (Vol. 1, 1972; Vol. 2, 1973)

[18] S. Kobayashi \& K. Nomizu: Foundations of Differential Geometry, Interscience, New York (Vol. 1, 1963; Vol. 2, 1969)

[19] R. Abraham \& J.E. Marsden: Foundations of Mechanics, $2^{\text {nd }}$ edition, BenjaminCummings, Reading 1978.

[20] R.C. Gunning: Lectures on Vector Bundles over Riemann Surfaces, Princeton University Press, Princeton 1967. 
[21] M. Forger \& S. Romero: Covariant Poisson Brackets in Geometric Field Theory, Commun. Math. Phys. 256 (2005) 375-410.

[22] M. Grassi: Polysymplectic Spaces, s-Kähler Manifolds and Lagrangian Fibrations, arXiv: math.DG/0006154v1.

[23] J. Martinet: Sur les Singularités des Formes Différentielles, Ann. Inst. Four. 20 (1970) 95-178. 